\title{
A novel method to obtain three- dimensional urban surface temperature from ground-based thermography
}

Article

Accepted Version

Creative Commons: Attribution-Noncommercial-No Derivative Works 4.0

Morrison, W., Kotthaus, S., Grimmond, C. S. B., Inagaki, A., Tin, T., Gastellu-Etchegorry, J.-P., Kanda, M. and Merchant, C. J. (2018) A novel method to obtain three-dimensional urban surface temperature from ground-based thermography. Remote Sensing of Environment, 215. pp. 268-283. ISSN 0034-4257 doi: https://doi.org/10.1016/j.rse.2018.05.004 Available at https://centaur.reading.ac.uk/77577/

It is advisable to refer to the publisher's version if you intend to cite from the work. See Guidance on citing.

To link to this article DOI: http://dx.doi.org/10.1016/j.rse.2018.05.004

Publisher: Elsevier

All outputs in CentAUR are protected by Intellectual Property Rights law, including copyright law. Copyright and IPR is retained by the creators or other copyright holders. Terms and conditions for use of this material are defined in the End User Agreement. 


\section{CentAUR}

Central Archive at the University of Reading

Reading's research outputs online 


\title{
A novel method to obtain three-dimensional urban surface temperature from ground-based thermography
}

William Morrison $^{1 *}$, Simone Kotthaus ${ }^{1,2}$, C.S.B. Grimmond ${ }^{1}$, Atsushi Inagaki ${ }^{3}$, Tiangang Yin ${ }^{4}$, Jean-Philippe GastelluEtchegorry $^{5}$, Manabu Kanda ${ }^{3}$, Christopher J. Merchant ${ }^{1,6}$

\author{
${ }^{1}$ Department of Meteorology, University of Reading, Earley Gate, Reading, RG6 6BB, UK \\ ${ }^{2}$ Institut Pierre Simon Laplace, France \\ ${ }^{3}$ Tokyo Institute of Technology, Japan \\ ${ }^{4}$ Center for Environmental Sensing and Modelling, Singapore-MIT Alliance for Research and Technology \\ ${ }^{5}$ CESBIO, Toulouse University (CNRS, CNES, IRD, UPS), Toulouse, France \\ ${ }^{6}$ National Centre for Earth Observation, University of Reading, UK \\ *Corresponding author: w.morrison@pgr.reading.ac.uk
}

Keywords: Ground-based thermography; Thermographic camera modelling; Image classification; Upwelling longwave radiation; Urban meteorology; COSMO; DART; Sensor view modelling

\section{Abstract}

Urban geometry and materials combine to create complex spatial, temporal and directional patterns of longwave infrared (LWIR) radiation. Effective anisotropy (or directional variability) of thermal radiance causes remote sensing (RS) derived urban surface temperatures to vary with RS view angles. Here a new and novel method to resolve effective thermal anisotropy processes from LWIR camera observations is demonstrated at the Comprehensive Outdoor Scale MOdel (COSMO) test site. Pixel-level differences of brightness temperatures reach $18.4 \mathrm{~K}$ within one hour of a 24-h study period. To understand this variability, the orientation and shadowing of surfaces is explored using the Discrete Anisotropic Radiative Transfer (DART) model and Blender three-dimensional (3D) rendering software. Observed pixels and the entire canopy surface are classified in terms of surface orientation and illumination. To assess the variability of exitant longwave radiation $\left(M_{L W}\right)$ from the 3D COSMO surface $\left(M_{L W}^{3 D}\right)$, the observations are prescribed based on class. The parameterisation is tested by simulating thermal images using a camera view model to determine camera perspectives of $M_{L W}^{3 D}$ fluxes. The mean brightness temperature differences per image (simulated and observed) are within $0.65 \mathrm{~K}$ throughout a 24 -h period. Pixel-level comparisons are possible with the high spatial resolution of $M_{L W}^{3 D}$ and DART camera view simulations. At this spatial scale $(<0.10 \mathrm{~m})$, shadow hysteresis, surface sky view factor and building edge effects are not completely resolved by $M_{L W}^{3 D}$. By simulating apparent brightness temperatures from multiple view directions, effective thermal anisotropy of $M_{L W}^{3 D}$ is shown to be up to $6.18 \mathrm{~K}$. The developed methods can be extended to resolve some of the identified sources of sub-facet variability in realistic urban settings. The extension of DART to the interpretation of ground-based RS is shown to be promising.

List of symbols and acronyms [units]

\begin{tabular}{|c|c|}
\hline$\beta, \phi, \omega$ & Euler angles describing a sequence of rotations within the $\left(x_{c}, y_{c}, z_{c}\right)$ coordinate frame \\
\hline BOA & Bottom of atmosphere \\
\hline BRF & Bidirectional reflectance factor \\
\hline $\mathrm{C}$ & Non-specific camera \\
\hline COSMO & COmprehensive urban Scale MOdel \\
\hline$d_{F P A}$ & Camera focal plane array size $[\mathrm{mm}]$ \\
\hline DART & Discrete Anisotropic Radiative Transfer model (Gastellu-Etchegorry et al., 2012) \\
\hline DSM & Digital surface model \\
\hline$\varepsilon$ & Emissivity \\
\hline$E_{L W}^{\downarrow}$ & Broadband longwave radiation flux (irradiance) downward from sky [W m $\left.\mathrm{m}^{-2}\right]$ \\
\hline$E_{S W}^{\downarrow}$ & Broadband shortwave radiation flux (irradiance) downward from sky [ $\left.\mathrm{W} \mathrm{m}^{-2}\right]$ \\
\hline$E_{S W, \text { dir }}^{\downarrow}$ & Broadband direct shortwave radiation flux (irradiance) downward from sky [ $\mathrm{W} \mathrm{m}^{-2}$ ] \\
\hline$F$ & Camera focal length $[\mathrm{mm}]$ \\
\hline$f$ & Fraction \\
\hline FOV & Field of view \\
\hline FPA & Focal plane array \\
\hline$i$ & Nonspecific surface class \\
\hline IFOV & Instantaneous field of view \\
\hline IP & Image plane \\
\hline$\lambda$ & Wavelength $[\mu \mathrm{m}]$ \\
\hline LW & Longwave \\
\hline LWIR & Longwave infrared \\
\hline$M_{L W}$ & Broadband longwave radiation flux (exitance) from a surface $\left[\mathrm{W} \mathrm{m}^{-2}\right]$ \\
\hline$M_{L W}^{3 D}$ & $\begin{array}{l}\text { Broadband longwave radiation flux (exitance) from discrete points of an urban surface, resolved in 3D [W } \\
\mathrm{m}^{-2} \text { ] }\end{array}$ \\
\hline$M_{L W}^{c a m}$ & Camera derived broadband longwave radiation flux (exitance) $\left[\mathrm{W} \mathrm{m}^{-2}\right]$ \\
\hline$M_{L W}^{c a n}$ & Non-specific broadband longwave radiation flux (exitance) from urban canopy elements [ $\mathrm{W} \mathrm{m}^{-2}$ ] \\
\hline
\end{tabular}




\begin{tabular}{|c|c|}
\hline$M_{L W}^{\uparrow}$ & Broadband longwave radiation flux (exitance) upward from ground [ $\left.\mathrm{W} \mathrm{m}^{-2}\right]$ \\
\hline$M_{L W}^{R S}$ & Nonspecific (e.g. satellite) remote sensing derived broadband longwave radiation flux (exitance) $\left[\mathrm{W} \mathrm{m}^{-2}\right]$ \\
\hline$M_{S W}^{\uparrow}$ & Broadband upwelling shortwave radiation flux (exitance) upward from ground [ $\left.\mathrm{W} \mathrm{m}^{-2}\right]$ \\
\hline$M_{S W}$ & Shortwave radiant flux (exitance) from a MW surface element $\left[\mathrm{W} \mathrm{m}^{-2}\right]$ \\
\hline MW & Model world \\
\hline$O$ & Origin of model world domain coordinate frame \\
\hline$O_{c}$ & Origin of camera intrinsic coordinate frame \\
\hline$P$ & Camera principle point \\
\hline$\phi$ & Zenith angle \\
\hline$\Psi^{\text {sky }}$ & Sky view factor \\
\hline$\Psi^{\text {can }}$ & Canopy view factor \\
\hline$R$ & Camera rotation parameters \\
\hline RGB & Red, green, blue \\
\hline RW & Real world \\
\hline$s$ & Camera pixel scaling factor \\
\hline$S$ & Triangle face of vector model \\
\hline SW & Shortwave \\
\hline$t$ & Camera translation parameters \\
\hline$\tau$ & Transmissivity \\
\hline$T_{b}$ & Brightness temperature $[\mathrm{K}]$ \\
\hline$T_{b}^{c a m}$ & Camera derived brightness temperature $[\mathrm{K}]$ \\
\hline TOA & Top of atmosphere \\
\hline$T_{S}$ & Thermodynamic surface temperature $[\mathrm{K}]$ \\
\hline$T_{S}^{3 D}$ & Thermodynamic surface temperature at discrete points of an urban surface, resolved in $3 \mathrm{D}[\mathrm{K}]$ \\
\hline$V x$ & Voxel (a volume element) \\
\hline$V x^{S}$ & Voxel intersected by a digital surface model element (surface voxel) \\
\hline$X, Y, Z$ & Model world domain coordinate frame \\
\hline$x, y$ & Camera pixel coordinate frame \\
\hline$x_{c}, y_{c}, z_{c}$ & Camera intrinsic coordinate frame \\
\hline$z_{c}$ & Camera principle axis \\
\hline
\end{tabular}

\section{Introduction}

Urban surface temperature $\left(T_{s}\right)$ plays a significant role in the urban surface energy balance as it is central to longwave radiation (LW), turbulent sensible heat and storage heat fluxes. Remote sensing (RS) methods have the potential to provide $T_{S}$ at large spatial scales for understanding exchanges of sensible heat (e.g. Voogt and Grimmond, 2000; Xu et al., 2008), the thermal comfort of city dwellers (Thorsson et al., 2004), and the urban surface heat island phenomenon (Huang et al., 2016; Kato and Yamaguchi, 2005; Roth et al., 1989). Two major challenges of urban thermal RS observations relate to the complex three-dimensional (3D) urban surface form and material heterogeneity, both causing large spatiotemporal variability of $T_{S}$ (Voogt and Oke, 2003). Spatiotemporal variability of $T_{S}$ is influenced by the relative orientation of surfaces to the sun during the day, and sky at night (Voogt and Oke, 2003). The diversity of thermal and radiative properties of surface materials causes additional variability (Voogt, 2008). What results is a directional variability, or an effective thermal anisotropy (Krayenhoff and Voogt, 2016), of broadband longwave radiation $\left(M_{L W}, \mathrm{~W} \mathrm{~m}^{-2}\right)$ from the urban canopy surface. The anisotropic behavior of urban canopies is defined as 'effective' to differentiate from thermal anisotropy exhibited by individual surface components (Voogt and Oke, 1998). Effective thermal anisotropy clearly affects satellite measured radiance, which is indicative of satellite derived longwave radiation $\left(M_{L W}^{R S}\right.$. As a result, the apparent $T_{S}$ can vary depending on view direction. $M_{L W}^{R S}$ can be described by:

$$
M_{L W}^{R S}=\sum_{i}^{n} M_{L W, i} f_{i}
$$

where $M_{L W, i}$ is the exitant broadband longwave radiation from a given canopy surface element $i$ that comprises fraction $f$ of the instrument field of view (FOV). Out of the total number of canopy surface elements $n, M_{L W, i}$ may be unique due to the highly variable radiative properties associated with its surface temperature, emissivity $(\varepsilon)$ and contributions from longwave reflections. $M_{L W}^{R S}$ is also sensitive to urban canopy geometry and to the specific view angle within each image swath. These factors combine to form a view angle specific $f_{i}$ which translates to a view angle specific value of $M_{L W}^{R S}$. For example, $f_{i}$ for roof and tree tops is generally overemphasised within $M_{L W}^{R S}$ for urban areas (Roth et al., 1989). Corrections of effective thermal anisotropy are critical when retrieving high-quality $T_{s}$ products for urban environments at large spatial scales from satellite-derived $M_{L W}^{R S}$.

The impact of effective thermal anisotropy on $M_{L W}^{R S}$ has been studied using various observation and modelling techniques. Observations from airborne platforms (e.g. Lagouarde et al., 2004; Sugawara and Takamura, 2006; Voogt and Oke, 1998) allow highly variable view angles at scales representative of satellite pixel resolutions $(100 \mathrm{~m}-1 \mathrm{~km})$. However, cost and air traffic restrictions usually limit these to short-term research campaigns. As obtaining different view angles requires multiple flyovers (i.e. difficult to conduct simultaneously), sequential flyovers with one aircraft may temporally confound results. Thus, the directional variability of $M_{L W}^{R S}$ at a micrometeorological timeframe (sub-hourly) for energy exchange 
processes (Christen et al., 2012) may be unresolved. Ground-based RS observations are interesting in that $M_{L W}$ can be resolved at high temporal resolutions (e.g. Christen et al., 2012) while resolving the individual facet (e.g. roof, wall) and sub-facet scale classes of $M_{L W, i}$ that constitute the structural and radiative characteristics of the urban canopy. For groundbased RS, a challenge is to sample enough facets representative of the complete 3D urban canopy at any one time. A single ground-based measurement provides a highly directional sample at high spatial resolution. Several ground-based sensors are required to sample facets of all orientations, unless a single ground-based sensor is operated on a rotating (e.g. Adderley et al., 2015) or mobile (e.g. Voogt and Oke, 1997) platform. As satellite-based RS is also inherently biased by FOV, it is important to be able to understand the nature of this bias.

Modelling can further help resolve the contribution of sub-facet scale variability of $M_{L W}$ on effective thermal anisotropy. The nature of effective thermal anisotropy and $M_{L W}^{R S}$ can be understood under constrained conditions at high temporal and spatial resolutions. Therefore, modelling is considered key to progress (Voogt, 2008; Voogt and Oke, 2003). Approaches typically involve a parameterisation of surface geometry, an energy balance model prescription of surface temperature and sensor view modelling of $M_{L W}^{R S}$ to resolve $M_{L W, i}$ and $f_{i}$ (eqn. 1) for a given surface-sensor viewing geometry. Surface temperatures can be prescribed from 2D (Kusaka et al., 2001; Sugawara and Takamura, 2006; Voogt, 2008), 2.5D infinite street canyon (e.g. Lagouarde et al., 2010) and 3D (Krayenhoff and Voogt, 2007, 2016; Soux et al., 2004) energy balance simulations to estimate $M_{L W, i}$ (eqn. 1) at facet (e.g. surface orientation, roof, ground) or sub-facet (e.g. insolation, material) scales.

Few sensor-view modelling studies exist that prescribe $M_{L W, i}$ from observations at facet and sub-facet scale, despite this complementing and constraining energy balance simulations. Classifying surfaces within ground-based RS source areas poses challenges because of the potentially diverse viewing geometries, complex 3D urban canopy structure, and low resolution of longwave infrared (LWIR) camera imagery. Previously, the spatial frequency distributions of $M_{L W}$ determined by ground-based LWIR imagery were used to infer canopy surface classes (e.g. Voogt and Grimmond, 2000) or surface classes were manually identified and extracted (e.g. Voogt, 2008). Manual approaches based on broadband thermal imagery are limited when the temperature contrast between facets is low (because of orientation or material properties). Information at multiple wavelengths can be valuable to improve classification. With maturing of sensor view modelling, it is becoming a powerful tool to objectively classify surface elements captured by RS imagery. Previous studies interpreting ground-based LWIR imagery have determined per-pixel path lengths for atmospheric correction of observations from on top of a high-rise building in Berlin (Meier et al., 2011). The SUM surface-sensor-sun model (Soux et al., 2004) enables sensor view modelling of $M_{L W, i}$ prescribed from observations, limited to urban surface geometry resolved as regular arrays of rectangular shaped buildings. Studies using SUM have prescribed temperatures intermittently (e.g. Voogt, 2008) from ground-based and airborne platforms observations. 3D rendering and editing software and a 3D vector model have facilitated the classification of ground-based LWIR imagery in a suburban area in Vancouver (Adderley et al., 2015). Here, classified temperature 'textures' were gap-filled to enable extrapolation across the 3D vector model as a complete brightness temperature product for sensor view modelling of hemispherical radiometer measurements using a single LWIR camera on a rotating mast.

In the current study, a flexible observational and modelling approach is developed to prescribe $M_{L W}$ from broadband longwave radiation fluxes derived from static ground-based LWIR camera observations. A 3D distribution of exitant broadband longwave radiation $\left(M_{L W}^{3 D}, \mathrm{~W} \mathrm{~m}^{-2}\right)$ is constructed from observations. The approach involves a novel method to classify each camera image. Pixels within each image are associated with a specific surface class prior to observations being extrapolated to all urban canopy surface elements in 3D. A 'model world' (MW) is used to process and interpret observations which enables 'real world' (RW) surfaces to be related to each camera image by camera view modelling. It provides a robust and quantitative method to interpret observations. Surface class $i$ is determined in 3D space [i.e. $i(X, Y, Z)]$ and is then accurately mapped to the $2 \mathrm{D}(x, y)$ coordinates of a camera image plane (IP) [i.e. $i(x, y)]$.

Unique here is the camera view modelling used to interpret observations, as surface classes are determined at high temporal and spatial resolution using surface geometry and shortwave (SW) radiative characteristics for each time step. This is designed to ensure all canopy surfaces are always accounted for when extrapolating observations over the 3D urban surface. A potential constraint of highly directional ground-based measurements is turned to an advantage by positioning two cameras at opposing view angles. This permits a combined observational source area representative of all surface classes that constitute the 3D urban surface. Extrapolated observations are compared with original camera imagery. This is done by projecting the extrapolated observations through the perspective of simulated cameras, with modelled perspectives matching those seen by the original camera imagery. This approach is unique in that the modelled perspectives are shown to reproduce the perspectives of the original imagery at pixel level and to a high degree of accuracy. Extrapolated observations have potential as a tool for further sensor view modelling to explore the impact of effective thermal anisotropy on directionally variable $M_{L W}^{R S}$ products for any given surface-sensor configuration.

The observational setup (Section 2.1, Section 2.2), the classification methods (Section 2.3, Section 2.4) and extrapolation (Section 3) of observations are introduced. Results (Section 4) include evaluation of proposed methods and demonstrate their benefits for application in urban RS. It is concluded (Section 5) that the detailed modelling approach provides a valuable tool for future studies in real city settings. 


\section{Methods}

LWIR camera observations are interpreted and estimated as $M_{L W}^{3 D}$ in a MW environment (Figure 1). Two LWIR cameras (Section 2.2) were installed on ground-based platforms above an urban test site (treated here as the RW, Section 2.1) to capture spatial and temporal variability of $M_{L W}$ that is representative of $M_{L W}^{3 D}$. The MW surface geometry (Section 2.3) and camera view (Section 2.4) components enable extrapolation of RW observations to $M_{L W}^{3 D}$ (Section 3).

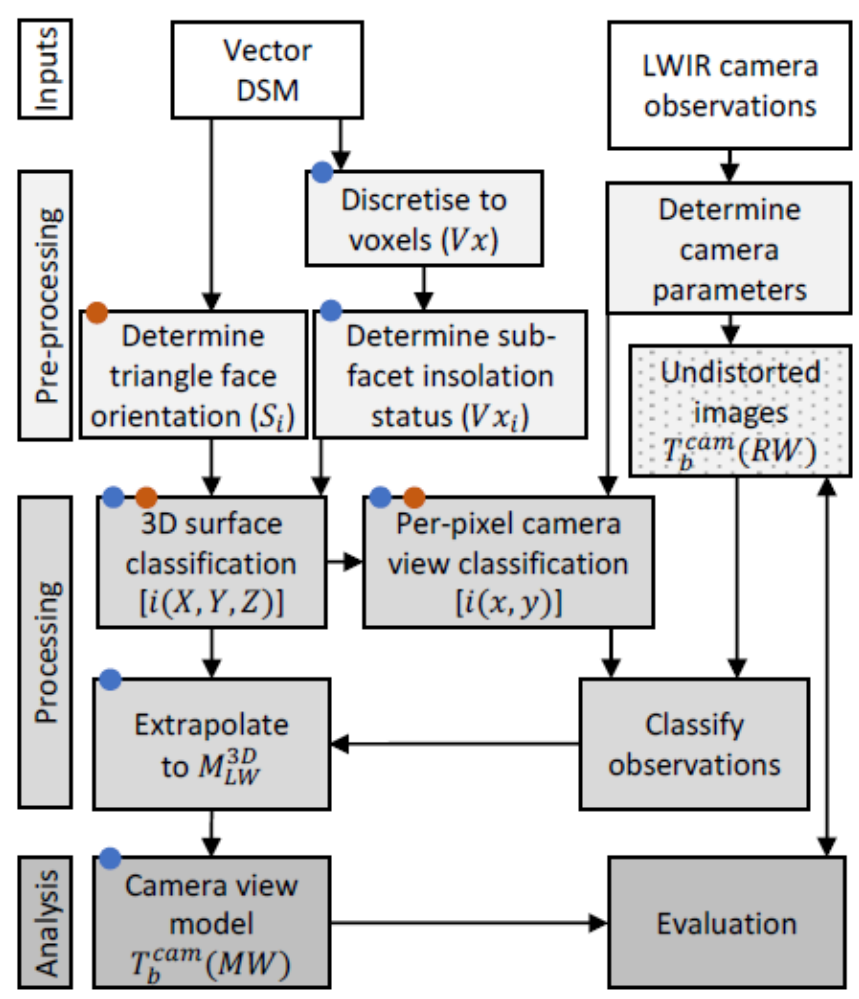

Figure 1. Flow chart of procedures to estimate and evaluate exitant broadband longwave radiation prescribed across an urban canopy $\left(M_{L W}^{3 D}\right)$ using ground based longwave infrared (LWIR) imagery coupled with camera view and $3 D$ modelling techniques. See list of symbols and acronyms for all other definitions.

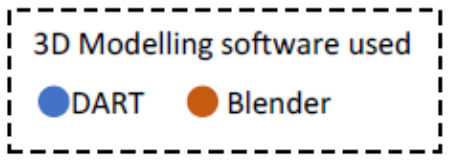

\subsection{Real world site}

The COSMO site (Kanda et al., 2007) is an outdoor scale model of an urban canopy. It occupies an area of $100 \mathrm{~m} \times 50 \mathrm{~m}$ (Figure 2) at the Nippon Institute of Technology, Saitama prefecture, Japan (South East corner: $36^{\circ} 1^{\prime} 36.42^{\prime \prime} \mathrm{N}, 139^{\circ} 42^{\prime}$ $18.45^{\mathrm{F}} \mathrm{E}$ ). The simple repeating geometry consists of $1.5 \mathrm{~m}$ cubic concrete blocks (with $0.1 \mathrm{~m}$ thick walls) with an even $1.5 \mathrm{~m}$ spacing (Figure 2d). The long axis for the $32 \times 16$ blocks is oriented $49^{\circ}$ west of true North (Figure 2a). For simplicity, the 'building' walls are referred to hereafter by their nearest cardinal direction relative to COSMO long axis orientation: $\mathrm{S}\left(229^{\circ}\right), \mathrm{E}\left(139^{\circ}\right), \mathrm{N}\left(49^{\circ}\right)$, and $\mathrm{W}\left(319^{\circ}\right)$. Obviously, this deviation from the true cardinal directions impacts shading patterns and related surface warming effects. All surfaces are made of the same concrete, painted grey (albedo $=$ $0.1, \varepsilon_{7-13 \mu m}=0.89$; Kawai et al. 2007). Surface weathering effects (Figure 2c) are likely to affect the radiative characteristics, but the apparently random patterns are too small a spatial scale to be accounted for in this study.

\subsection{Real world Instrumentation}

Two Optris PI160 LWIR cameras (Optris GmbH, Germany) were installed on an aluminium lattice tower at $6.8 \mathrm{~m}$ and 7.0 $\mathrm{m}$ above ground level (Figure 2d) at opposing azimuth angles with oblique views of ground, roof and all cardinal facing surfaces. Cameras are defined as $\left(\mathrm{C}^{\text {north }}, \mathrm{C}^{\text {south }}\right.$ ) based on their azimuthal view angle (Figure $2 \mathrm{~b}$ ). The horizontal and vertical pixel resolution of the cameras ranges from approximately $0.030 \mathrm{~m} \mathrm{x} 0.043 \mathrm{~m}$ to $0.079 \mathrm{~m} \times 0.111 \mathrm{~m}$. The small, lightweight industrial grade cameras use uncooled microbolometer technology, with $25 \mu \mathrm{m}$ x $25 \mu \mathrm{m}$ bolometer elements arranged as a $160 \times 120$ focal plane array (FPA). With multiple cameras, multiple view angles (e.g. Figure $2 \mathrm{~b}$ ) can be sampled simultaneously in a static setup (cf., rotating one sensor, Adderley et al., 2015; or vehicle traverses, Voogt and Oke, 1997). The instrument outputs digital number values for each microbolometer pixel. These values relate to at-sensor broadband $7-13 \mu \mathrm{m}$ radiance and are radiometrically calibrated by the manufacturer to brightness temperatures $\left[T_{b}^{c a m}(x, y)\right]$ using black body reference measurements. The per-pixel broadband longwave radiation flux $\left[M_{L W}^{c a m}(x, y)\right]$ is related to $T_{b}^{c a m}(x, y)$ by Stefan-Boltzmann law:

$$
M_{L W}^{c a m}(x, y)=\sigma T_{b}^{c a m}(x, y)^{4}
$$

with $\sigma$ the Stefan-Boltzmann constant $\left(5.67 \times 10^{-8} \mathrm{~W} \mathrm{~m}^{-2} \mathrm{~K}^{-4}\right)$. The temperature resolution is $0.1 \mathrm{~K}$ and the manufacturer's specified accuracy is $2{ }^{\circ} \mathrm{C}$ at ambient temperatures $23 \pm 5^{\circ} \mathrm{C}$ (Optris $\mathrm{GmbH}$, Germany). Although images can be captured 
at $120 \mathrm{~Hz}$, for this study images recorded every $60 \mathrm{~s}$ are used to reduce data overhead but capture temporal variability of $M_{L W}^{c a m}$ caused by transient surface shadowing. The manufacturer specified camera horizontal and vertical FOV is $41^{\circ} \mathrm{x} 31^{\circ}$. Observations were taken between 2014/06/16 and 2014/09/26. In this paper, the focus is on a predominantly clear-sky day (2014/08/02). Both cameras were connected to the same field laptop for data acquisition via USB using the Optris PI connect software.

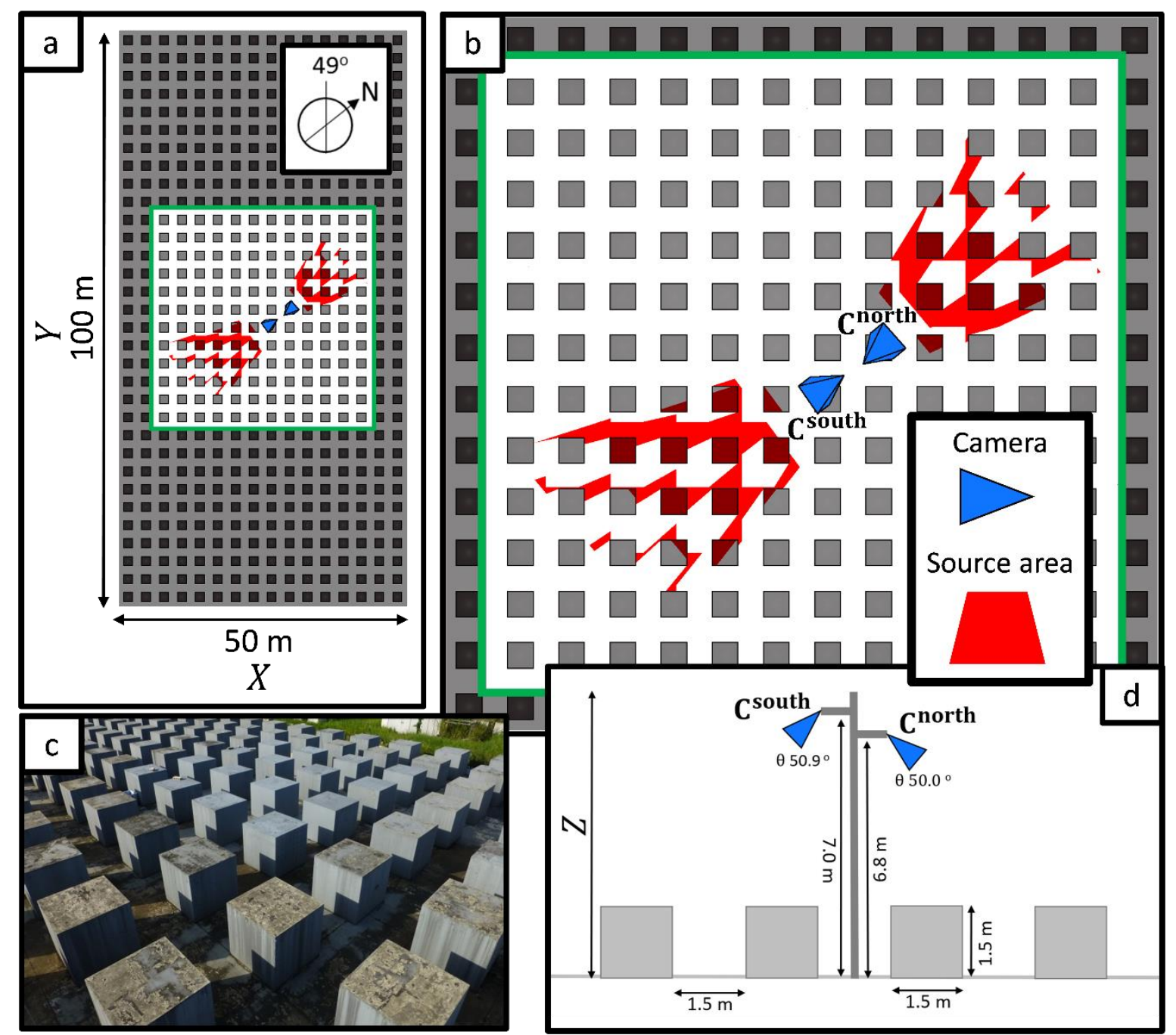

Figure 2. COSMO test site and longwave infrared (LWIR) camera observational setup: (a) test site domain (plan view) with focus area (green box); (b) focus area with LWIR camera $\left(C^{\text {south }}, C^{\text {north }}\right)$ locations and approximate orientations (blue) and source areas (red) for camera field of view; (c) north-west facing oblique visible image taken near the $C^{\text {north }}$ camera location and perspective (d) vertical cross section of building array (grey squares) showing instrument tower and camera geometry.

Multiple internal processing steps need to be considered to achieve radiometrically calibrated measurements from LWIR cameras. Uncooled microbolometer calibration and measurement processes are reviewed by Budzier and Gerlach (2015). Here the quality control steps undertaken are presented.

After a camera is sited, the FPA requires a "warm up" period to allow the current induced self-heating of the sensor elements to stabilise (Vollmer and Möllmann, 2010) prior to measurements. As laboratory testing found up to $2 \mathrm{~h}$ warm up period is required depending on target and camera body temperature conditions (cf. manufacturer's recommended 10 mins), data prior to this are excluded. To correct for changes in the contribution of interior radiance incident on the FPA due to any change in the camera body temperature resulting from ambient air temperature variability, a shutter inside the camera with assumed black body characteristics and of known temperature obscures the sensor before every measurement so that its emission is sampled. The cameras are fitted with aluminum covers (enclosure: $945 \mathrm{~mm}$ x $45 \mathrm{~mm}$ x $62 \mathrm{~mm}$ ). These are designed to prevent lens exposure to precipitation and any rapid, directional heating of the sensor body due to direct sun exposure.

\subsection{Model world site}

To interpret $M_{L W}^{c a m}$ for subsequent parameterisation and evaluation of $M_{L W}^{3 D}$, a MW is used. It has realistic surface-sensor geometry and processes contributing to variability in $M_{L W}$ exitant across the RW (COSMO) site. For a given RW point at $3 \mathrm{D}$ coordinates $(X, Y, Z)$, the RW radiative processes that determine $M_{L W}$ from the canopy surface $\left[M_{L W}^{3 D}(X, Y, Z)\right]$ are 
approximated by assuming Lambertian facets, first order scattering, isotropic sky thermal radiance, invariance of emissivity across a broadband of thermal wavelengths and invariance of emissivity with facet kinematic temperature, viz:

$$
\begin{aligned}
& M_{L W}^{3 D}(X, Y, Z)=\sigma T_{b}^{3 D^{4}}(X, Y, Z)=[1-\varepsilon(X, Y, Z)] \cdot \Psi^{\text {sky }}(X, Y, Z) \cdot E_{L W}^{\downarrow} \\
& +[1-\varepsilon(X, Y, Z)] \cdot \Psi^{\text {can }}(X, Y, Z) \cdot M_{L W}^{c a n} \\
& +\varepsilon(X, Y, Z) \cdot \sigma T_{s}^{3 D^{4}}(X, Y, Z) .
\end{aligned}
$$

where $\Psi^{s k y}$ and $\Psi^{\text {can }}$ are sky and canopy view factors (Johnson and Watson, 1984) that influence the radiant flux incident on RW point $(X, Y, Z) ; E_{L W}^{\downarrow}$ is broadband longwave irradiance from sky; $M_{L W}^{c a n}$ is broadband longwave radiation emitted from surrounding canopy elements; $T_{b}^{3 D}(X, Y, Z)$ and $T_{s}^{3 D}(X, Y, Z)$ are the surface brightness and kinematic temperatures for the given point; and $\varepsilon(X, Y, Z)$ the broadband surface emissivity for the given point. The COSMO test site (Figure 2) and observational period chosen enables eqn. 3 to be simplified: 1$)$ the homogeneous surface material allows $\varepsilon(X, Y, Z)$ to be treated as constant and isotropic, and 2) the high material emissivity reduces any variability in reflection contributions from $E_{L W}^{\downarrow}$ and $M_{L W}^{c a n} . E_{L W}^{\downarrow}$ is assumed isotropic in eqn. 3 as a simplification due to clear-sky conditions for the study date. The remaining factors determining $\operatorname{COSMO} M_{L W}^{3 D}(X, Y, Z)$ in eqn. $3\left(T_{S}^{3 D}, \Psi^{\text {sky }}\right.$ and $\left.\Psi^{\text {can }}\right)$ are highly variable across the site. To parameterise the variability of $M_{L W}^{3 D}$ in this paper, facets are classified by their orientation using Blender (Blender Foundation, 2016) and sub-facet insolation status (or shadow patterns) using the DART 3D radiative transfer model (Gastellu-Etchegorry et al., 2012) (Figure 1). Combining Blender (version 2.78) and DART (version 5.6.6, build v935) allows the 3D distribution of specific surfaces classes $[i(X, Y, Z)]$ to be determined across the site. $i(X, Y, Z)$ is prescribed with similarly classified observations to formulate $M_{L W}^{3 D}$.

Two spatial reference systems defined in the MW by DART and Blender facilitate the creation of $i(X, Y, Z)$ :

1) In both DART and Blender, the RW surface geometry is represented by a vector-based digital surface model (DSM) of triangles in a 3D mesh. This resolves surface geometry at a high level of detail (Gastellu-Etchegorry, 2008) which is not limited to simple geometry (e.g. Soux et al., 2004). A triangle face $(S)$ is the planar area between three vertices each with $X, Y, Z$ coordinates (Figure 3 ) with attribute $S_{i}$ a determinable facet-scale surface class.

2) In DART, the MW is discretised into voxels $V x$ of uniform size in a 3D raster format (see Figure 3; $\Delta X, \Delta Y, \Delta Z$; Yin et al., 2015). Surface voxels contain surface elements of the DSM $V x^{S}$ (Figure 3), whereas other voxels only contain atmosphere. Voxels enable radiative transfer processes to be calculated within DART at high (sub-facet scale; $<S$ ) resolution. Surface voxels (Figure 3) are used to track radiation emitted and intercepted by $S$ (Gastellu-Etchegorry, 2008), meaning MW geometry is resolved by the DSM during simulation. The prescribed surface temperature $\left(V x_{T_{S}}^{S}\right)$ and sub-facet-scale surface class information $[i(X, Y, Z)]$ are stored by surface voxels. Therefore, a surface voxel that occupies an area $\Delta X, \Delta Y, \Delta Z$ of the DSM stores surface class $V x_{i}^{S}(\Delta X, \Delta Y, \Delta Z)$ and temperature $V x_{T_{S}}^{S}(\Delta X, \Delta Y, \Delta Z)$ data. Simulated sources of emitted radiation can be from any combination of sun, upper atmosphere, $V x^{S}$ and atmosphere voxels. Here, sun angle and insolation are modelled by DART to determine $V x_{i}^{S}(\Delta X, \Delta Y, \Delta Z)$ for sunlit $\left[V x_{\text {sunlit }}^{S}(\Delta X, \Delta Y, \Delta Z)\right]$ and shaded $\left[V x_{\text {shaded }}^{S}(\Delta X, \Delta Y, \Delta Z)\right]$ elements of the MW surface at a spatial resolution of $\Delta X, \Delta Y, \Delta Z=0.04 \mathrm{~m}$ which is representative of the $\mathrm{RW}$ observation spatial resolution (Section 2.2)

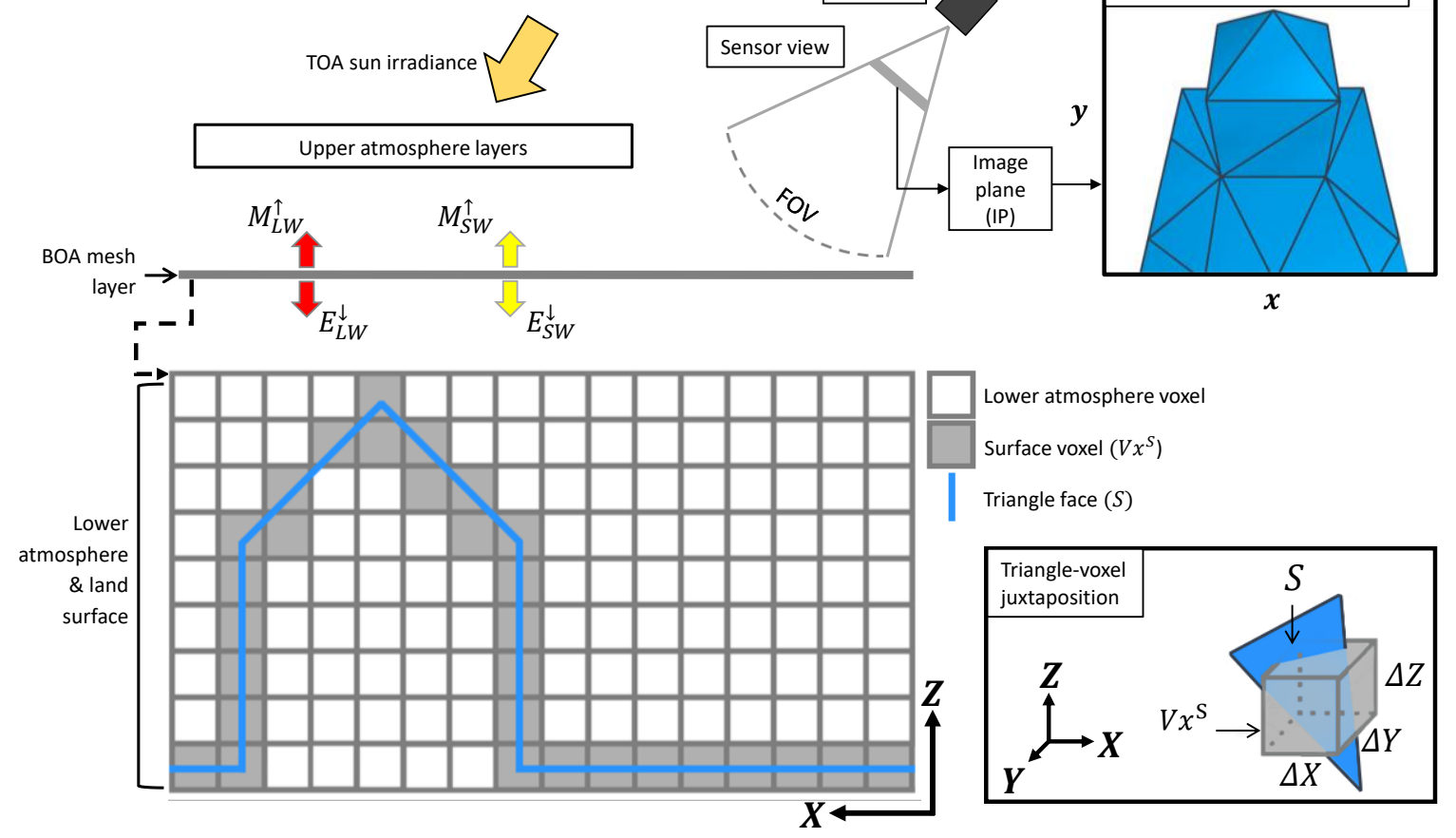

Figure 3. Surface representation and interaction in the 'model world' $(M W)$ is defined by triangle face (S) and voxel (Vx) elements, with camera view modelling to simulate camera image plane (IP). See table and text for symbol and acronym definitions. 
DART can simulate radiative transfer processes in the visible to LWIR regions of the electromagnetic spectrum (Yin et al., 2015 ) in the atmosphere and any urban or natural landscape. Individual rays are tracked along discrete directions within angular cones (Yin et al., 2013). Landscape, or 'bottom of atmosphere' (BOA), illumination is due to direct and diffuse sun radiation $\left(E_{S W}^{\downarrow}\right)$ and $E_{L W}^{\downarrow}$. It is simulated as rays that flow from a horizontal BOA layer at the top of the landscape (Figure 3 ). The surface density of these illumination rays is $1 / D^{2}$, with $D$ the BOA illumination grid resolution. To simulate RW camera images taken above the BOA layer (Figure 3), rays that reach the BOA mesh layer are projected onto a simulated camera IP (Yin et al., 2015). A comprehensive description of DART including further functionality beyond the scope of this paper is provided by Gastellu-Etchegorry et al. (2015). DART camera image simulation specifics are detailed in Yin et al. (2015).

\subsubsection{Surface creation}

The DSM was created using Blender, based on the known site geometry (Section 2.1) and stored as a DART compatible "*.obj" wavefront data format. Here the metadata stored by this format for each triangle face includes facet orientation for the surface classification (Section 2.3.2). The DSM has the RW surface geometry (Figure 2) for the full site $(X=50 \mathrm{~m}, Y=$ $100 \mathrm{~m}, Z=1.5 \mathrm{~m}$ ), discretised into surface voxels using the MW definition of DSM - voxel interaction (Figure 3) at a resolution of $\Delta X=\Delta Y=\Delta Z=0.04 \mathrm{~m}$. The $X$ axis of the voxel array is aligned with the $X$ axis of the DSM (Figure 2).

\subsubsection{Surface classification}

The spatial and temporal class characteristics (orientation, surface insolation state) allow a dynamic high spatial resolution $3 \mathrm{D}$ classification of the MW surface as $i(X, Y, Z)$.

DART stores local incident and intercepted radiation in two different ways. It stores the upward directional radiance per surface element of the landscape, for simulating RS measurements. Also, the landscape 3D radiative budget is stored: irradiance and exitance per voxel upper face; and the radiation that is intercepted, absorbed and emitted per voxel. Here, the DART simulated 3D radiative budget is used to determine the sunlit or shaded status of $V x_{i}^{S}(X, Y, Z)$ through time, where $i=$ sunlit or $i=$ shaded. The sunlit and shaded areas of the MW are resolved at the voxel size $(\Delta X=\Delta Y=\Delta Z=0.04$ $\mathrm{m}$, Section 2.3.1). Direct downwelling SW radiation $\left(E_{S W, \text { dir }}^{\downarrow}\right)$ is simulated with solar angles calculated using NOAA solar calculator equations (NOAA, 2016). Here, DART tracks BOA rays (mesh cell size $D=0.02 \mathrm{~m}$ ) with radiant flux density $E_{S W}(\Omega, \theta, \phi)\left(\mathrm{W} \mathrm{m}^{-2}\right)$ along solid angle $\Omega(\mathrm{sr})$ with direction $(\theta, \phi)$ until incident on a DSM triangle. Hence, each triangle intercepted $E_{S W}(\Omega, \theta, \phi)$ is stored for the voxel that occupies the $3 \mathrm{D}$ space of the triangle (Figure 3 ) which across the entire scene produces a $3 \mathrm{D}$ array of voxels with values of intercepted irradiance $\left(\mathrm{W} \mathrm{m}^{-2}\right)$. If a surface voxel has stored no direct solar irradiance (i.e. cloud, night or building obstruction) then the voxel is classified as shaded $\left(V x_{i=s h a d e d}^{S}\right)$, otherwise it is sunlit $\left(V x_{i=\text { sunlit }}^{S}\right)$. If RW geometry were more complex, the classes could be split into discrete or binned values of irradiance intercepted by a MW surface.

The surface orientation attribute of each triangle face $S$ (Section 2.3.1) is one of the four cardinal orientations relative to north (e.g. $i=$ east) or horizontal orientations (e.g. $i=$ roof). Blender is used to determine the smallest angular difference between a triangle normal and the normal of each orientation. Once classified, the DSM is used to determine the orientation of sunlit or shaded voxels. Any sunlit or shaded surface voxel intersected by $S_{i}$ is classified as a sunlit or shaded voxel with orientation class $i$ (e.g. $\left.V x_{i=\text { roof, sunlit }}^{S}(X, Y, Z)\right)$.

\subsection{Model world instrumentation}

MW 'instrumentation' is used to classify each pixel of a RW camera observation by camera view modelling and to perform camera view modelling of $M_{L W}^{3 D}$ for a given RS view angle. Here, a MW 'instrument' is defined as the simulation of a RW camera perspective using camera view modelling. The RW camera images are classified at pixel level as $i(x, y)$ using basic pinhole cameras as the MW instruments. A basic pinhole camera has a rectilinear projection, meaning any straight lines in the MW domain are always projected as straight lines in the pinhole camera IP. A comprehensive description of this technique can be found in Hartley and Zisserman (2000). An overview of the steps taken to map a MW domain surface element with coordinates $(X, Y, Z)$ onto an IP with pixel coordinates $(x, y)$ is presented in Figure 4 . Common discrepancies between a theoretical and RW camera are highlighted, with methods given for the calibration of the lowresolution RW LWIR cameras to perform as a pinhole camera (Section 2.4.1). Methods used to apply the MW camera with the classified DSM (Section 2.4.3) are given along with uncertainties associated with the alignment error between RW and MW camera perspectives (Section 2.4.2).

All cameras have extrinsic and intrinsic parameters that determine the $(X, Y, Z) \rightarrow(x, y)$ coordinate transformation (Figure 4). Extrinsic parameters of rotation $(R)$ and translation $(t)$ describe the rigid transformation of a 3D coordinate frame to a $3 \mathrm{D}$ camera reference frame $(X, Y, Z) \rightarrow\left(x_{c}, y_{c}, Z_{c}\right)$ with new coordinate origin $O_{c}$ (Hartley and Zisserman, 2000; Heikkila and Silven, 1993). RW cameras with physically small, wide-angle lenses exhibit radial distortion, meaning image points are displaced radially in the IP. This type of projection is not comparable to that of an ideal pinhole camera. Here, the camera intrinsics and lens distortion parameters are defined together as the camera internal parameters, which must be estimated (Section 2.4.1). 


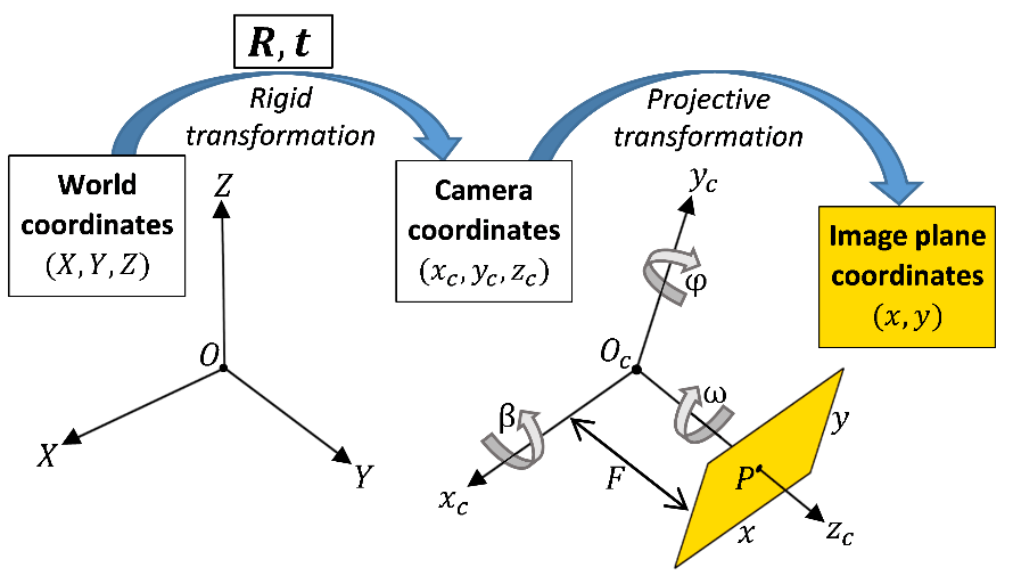

Figure 4. Coordinate and transformation definitions for 'model world' elements. $(X, Y, Z) \rightarrow\left(x_{c}, y_{c}, z_{c}\right)$ is the rigid transformation from the three-dimensional coordinate frame with origin $O$, to the three-dimensional camera coordinate frame with origin $O_{c}$ using camera extrinsic parameters of rotation $R$ and translation $t .\left(x_{c}, y_{c}, z_{c}\right) \rightarrow(x, y)$ is the projective transformation from camera coordinate frame to two-dimensional camera image plane (IP) frame (yellow). R represents a series of Euler angles $\beta, \phi$ and $\omega$ that define a sequence of rotations: first around the $x_{c}$-axis $(\beta)$, then around the $y_{c}$-axis $(\phi ')$ that has already been rotated by $\beta$, and finally around the $z_{c}$-axis ( $\omega$ ") that has already been twice rotated firstly by $\beta$ and then $\phi$ '(Heikkila and Silven, 1993). $t$ is a vector that describes the $M W$ origin $(O)$ as camera coordinate origin $\left(O_{c}\right)$. Intrinsic parameters of focal length $F$, pixel scale factor (eqn. 4) and principle point offset are used for the final projection of $3 D$ points onto the $2 D$ camera IP as $\left(x_{c}, y_{c}, z_{c}\right) \rightarrow(x, y)$. These parameters are determined by physical camera features including pixel size and the relative position of the IP to $O_{c}$. The point at which the principle axis $\left(z_{c}\right)$ intersects with the IP is the principle point $P$ (Hartley and Zisserman, 2000). For a pinhole camera, $P$ intersects at the centre of the IP. For RW cameras, the principle point offset describes the offset between $P$ and the centre of the IP which may arise from imperfections in the lens-FPA assembly. This results in a misalignment of the lens with the FPA (Clarke et al., 1998) and hence needs to be accounted for.

\subsubsection{Estimation of camera internal parameters}

To formulate a MW camera, extrinsic and internal parameters must be known or estimated. Extrinsic parameters of RW camera location and orientation are determined by on-site measurements. Internal parameters are required to match the RW image projection to a MW pinhole camera. A method is presented to experimentally estimate the internal parameters of a RW LWIR camera for correction of raw images to a rectilinear pinhole projection. The method requires known parameters of physical FPA size $\left(d_{F P A}, \mathrm{~mm}\right)$ and image resolution $\left(n_{p x}\right.$, pixels) which are obtained from instrument specifications.

Camera internals are determined using a $0.5 \mathrm{~m} \times 0.5 \mathrm{~m}$ polished steel plate $(\varepsilon \approx 0.02)$ populated by squares of masking tape $(\varepsilon \approx 0.95)$ to produce a planar calibration grid of $8 \times 7$ cells each $0.05 \mathrm{~m} \mathrm{x} 0.05 \mathrm{~m}$. This configuration allows a grid cell corner to be identified as $\left(X_{o}, Y_{o}, Z_{o}\right)$ in camera $(x, y)$ coordinates (Figure 4$)$. When placed outside on a clear or totally overcast day, the grid pattern can be observed in the LWIR due to the emissivity contrast between steel and masking tape. Images are taken until the grid has been captured by all parts of the LWIR camera IP at different rotations $(\sim 25$ images per camera). Captured images are processed with the Matlab camera calibration toolbox (Bouguet, 2015) to map each grid cell corner, $\left(X_{o}, Y_{o}, Z_{o}\right)$ to $(x, y)$ coordinates. Estimated camera extrinsic and internal parameters are used to transform the image using the Matlab toolbox. Grid corner points are again detected in this transformed image and compared to points projected onto the IP by the estimated extrinsic and internal parameters. This is an iterative process that stops when the error between detected and projected points is minimised in the least squares sense. The internal parameters applied to achieve this "best fit" between detected and projected points are assigned as the camera internal parameters enabling it to be treated as a pinhole camera.

The specified FOV (Section 2.2) decreases when images are transformed to pinhole projections (Table 1). The FOV is determined by obtaining the focal length $(F)$ from the calibrated pixel scaling factor $(s)$ and the known FPA size $(d)$ in the image $x$ or $y$ coordinate directions with known FPA resolution $\left(n^{p x}\right)$ in the image $x$ or $y$ coordinate directions:

$$
F=s\left(d_{F P A} / n^{p x}\right)
$$

which is related to the camera FOV (radians) in the image $x$ or $y$ coordinate direction by:

$$
F O V=2 \arctan \left(d_{F P A} / 2 F\right) \text {. }
$$

The derived internal parameters are used to re-map each pixel from each RW image using the nearest neighbour technique.

Table 1 Field of view of undistorted pinhole camera equivalent LWIR cameras used in the study. See text for methods. See Figure 2 for camera locations.

\begin{tabular}{c|c|c}
\multirow{2}{*}{ Camera ID } & \multicolumn{2}{c}{ Undistorted FOV } \\
& Horizontal $\left({ }^{\circ}\right)$ & Vertical $\left({ }^{\circ}\right)$ \\
\hline $\mathrm{C}^{\text {north }}$ & 41.4 & 31.6 \\
\hline $\mathrm{C}^{\text {south }}$ & 40.5 & 30.9 \\
\hline
\end{tabular}




\subsubsection{Reprojection error}

Assuming internal parameters have been accurately accounted for (Section 2.4.1), any misalignment between RW and MW camera perspectives depends on the prescribed MW camera extrinsic parameters of $(\beta, \phi, \omega)$ rotation $(R)$ and $(X, Y, Z)$ translation $(t)$ shown in Figure 4 . These parameters can be determined from RW measurements. An uncertainty in these measurements translates as an error in the MW camera perspective. A misalignment error based on estimates of uncertainty associated with on-site measurement of camera extrinsic parameters is calculated in root mean square error (RMSE) terms. Firstly, the extrinsic parameters for camera $C^{\text {south }}$ (Figure 2) measured on site are defined as "aligned" parameters. It is assumed that measurements of camera location (performed using a tape-measure) have an estimated measurement uncertainty of $0.1 \mathrm{~m}$ for each location axis. $(\beta, \phi)$ are estimated from azimuth and zenith angle measurements $(\theta, \phi)$ taken using a compass and protractor, respectively. Cameras are installed with no intentional rotation around the camera axis $(\omega)$. Each rotation has an assumed uncertainty of $1^{\circ}$. To quantify the impact of this assumed $R$ and $t$ measurement uncertainty, all possible permutations of these rotations and translations are determined at resolution of $\Delta^{\mathrm{o}}=0.5\left(\mathrm{e} . \mathrm{g}\right.$. $\left[\beta-1^{\mathrm{o}}\right] \rightarrow[\beta+$ $\left.\left.1^{\circ}\right], \Delta^{\mathrm{o}}=0.5\right)$ and $\Delta \mathrm{m}=0.05$ (e.g. $\left.[X-0.1 \mathrm{~m}] \rightarrow[X+0.1 \mathrm{~m}], \Delta \mathrm{m}=0.05\right)$. For each permutation, all roof vertices of the $\operatorname{DSM}(X, Y, Z)$ are updated with new MW coordinates $\left(X^{\prime}, Y^{\prime}, Z^{\prime}\right)$ by rotating and translating each DSM roof vertex around the camera origin $O_{c}$ (Figure 4 ) based on the permutation-specific $(R, t)$ values. The Euclidean distance $d$ between the original and updated vertices, where:

$$
d=\sqrt{\left(X^{\prime}-X\right)^{2}+\left(Y^{\prime}-Y\right)^{2}+\left(Z^{\prime}-Z\right)^{2}}
$$

is used to quantify the misalignment RMSE. Only roof vertices are analysed as occluded surfaces cannot be tracked from the camera perspective. With this degree of uncertainty, the maximum RMSE between all roof vertices within the camera FOV is $0.43 \mathrm{~m}$. A final adjustment of simulated $(R, t)$ extrinsic parameters is needed as this error is significant. This is challenging given that $(R, t)$ combine to give a high number of degrees of freedom. Adjustment is done using the experimentally derived $(R, t)$ parameters applied to a Blender pinhole camera perspective of the DSM. A RW camera image corrected to pinhole camera projection (Section 2.4.1) is made semi-transparent and then draped over the Blender camera FOV. The camera is then moved interactively around the DSM allowing $(R, t)$ to be manually adjusted as a supervised final alignment. A new alignment uncertainty when extrinsic parameters are manually adjusted is assumed $<$ $0.1^{\circ}$ for each rotation and $<0.05 \mathrm{~m}$ for each location parameter (RMSE $<0.06 \mathrm{~m}$ ). This method yields good alignment results when comparing the projected geometry for RW (Figure 5a, e) and MW (e.g. Figure 5b, c) imagery, which is further evaluated using high resolution digital camera imagery (Figure 6).

\subsubsection{Classification of camera images}

The classified MW surface (Section 2.3.2) is projected onto a MW camera IP to facilitate RW image classification. MW cameras are created using Blender and DART to simulate the RW camera per-pixel perspective of orientation (e.g. Figure 5b) and shadowing (e.g. Figure 5c) attributes, respectively.

The 3D rendering capabilities of Blender are used to classify images by surface orientation. All triangle faces of the DSM within an orientation class $i\left(S_{i}\right.$, Section 2.3.2) are assigned a colour with a specific RGB value. The DSM is then projected onto each MW camera IP to produce images with per-pixel RGB values related to each orientation class (Figure $5 \mathrm{~b}, \mathrm{f}$ ) which enables per-pixel surface classification as $i(x, y)$. The instantaneous field of view (IFOV) of some pixels within these images contain more than one surface class. This effect translates as pixels without a RGB value associated with a single class. Pixels with this characteristic are classified as "mixed". The radiometer boom within $\mathrm{C}^{\text {south }}$ observations (Figure 5a) is manually masked. Mixed and masked pixels are not included as part of any surface class.
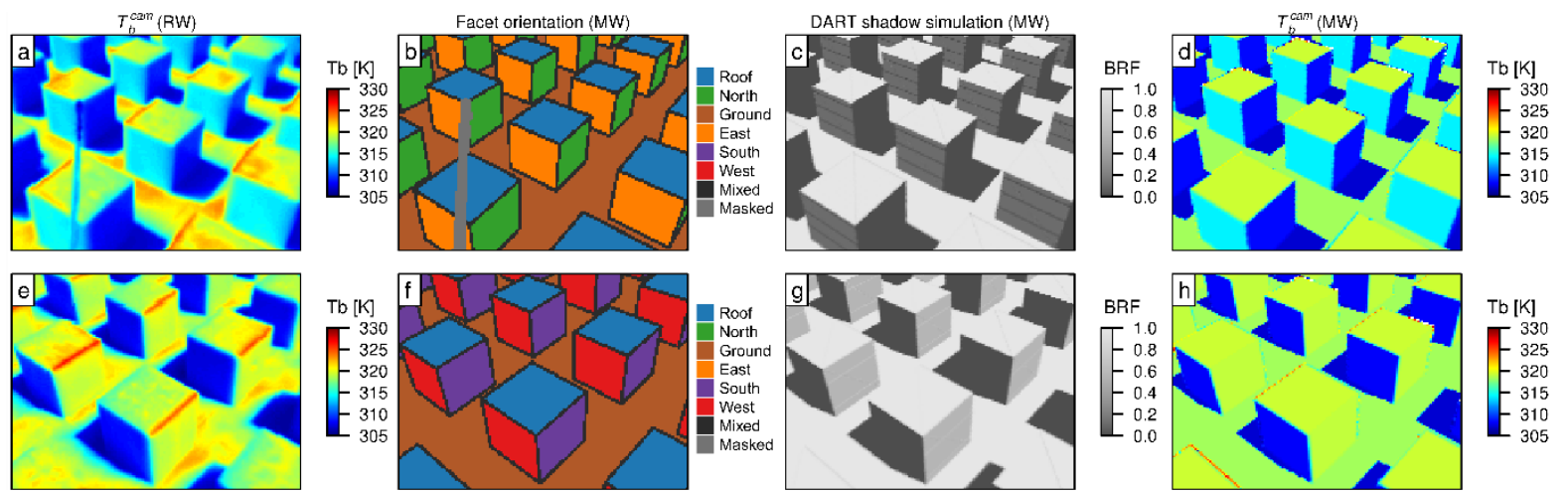

Figure 5. Observations, classification and extrapolation of results for Optris PI longwave infrared (LWIR) camera $(a-d) C^{\text {south }}$ and $(e$ - h) $C^{\text {north }}$ at COSMO test site (Figure 2, $2^{\text {nd }}$ August 2014 10:00 local standard time). (a, e) 'Real world' (RW) brightness temperature $\left(T_{b}\right)$ images; $(b, f)$ classification of per-pixel surface orientation using Blender 'model world' $(M W)$ camera view of the digital surface model (DSM) containing surface orientation information. Surface information is coded as per-class RGB textures projected onto a Blender camera for per-pixel identification of surface orientation. RGB values in the image not associated with an RGB texture class see more than one class and are classified as mixed pixels (black); $(c, g)$ classification of per-pixel surface shadowing derived by $D A R T$ shortwave scattering simulation and camera view model shown as shortwave bidirectional reflectance factor (BRF; 
Schaepman-Strub et al., 2006), dark pixels $(B R F=0)$ indicate no first order scattering from surface to camera; $(d, h)$ simulated $T_{b}$ thermographs: $T_{b}$ observations extrapolated to a $M W$ three-dimensional distribution based on surface classes (shown in $\left.b, c, f, g\right)$ reprojected onto a MW camera image plane simulated in DART, simulating 'real world' $(R W)$ camera observations $(a, e)$.

Sunlit and shaded pixels for each RW image are classified using the 3D distribution of sunlit and shaded surfaces from the DART simulation of direct downwelling SW radiation (Section 2.3.2). DART camera view modelling enables the 3D distribution of sunlit and shaded surfaces to be projected onto the MW camera perspective (Figure 5c, g). First order scattering of $E_{S W}(\Omega, \theta, \phi)$ from a surface is considered during the image classification, with DSM triangles being assigned as Lambertian reflectors in DART. Scattering from the surface occurs isotropically with exitance $M_{S W}^{\uparrow}\left(\mathrm{W} \mathrm{m}^{-2}\right)$ :

$$
M_{S W}^{\uparrow}=\int_{2 \pi} E_{S W}(\Omega, \theta, \phi) \cdot \cos \theta \cdot d \Omega .
$$

Due to first order scattering, any pixels of the DART MW camera with at-sensor radiance $>0 \mathrm{~W} \mathrm{~m}^{-2} \mathrm{sr}^{-1}$ originates from a sunlit voxel $\left(M_{S W}^{\uparrow}>0 \mathrm{~W} \mathrm{~m}^{-2}\right)$ intersected by a sunlit portion of the DSM. The IFOV of pixel $(x, y)$ with at-sensor radiance $>0 \mathrm{~W} \mathrm{~m}^{-2} \mathrm{sr}^{-1}$ is therefore classified as observing a sunlit surface (e.g. Figure $5 \mathrm{~b}$ ). Isolated pixels (i.e. no adjacent pixels of the same class) are reclassified as "mixed" as it is assumed there is insufficient spatial representation of the surface class from one pixel. The DART modelling of shadow distributions is evaluated in the RW using a Panasonic DMC-TZ31 digital camera image taken during clear sky daytime conditions (Figure 6a). The digital camera is assumed to exhibit pinhole camera characteristics. Shadow distributions across the image are then classified using a DART MW camera using manufacturer derived internal camera parameters. The illuminated surface geometry and distribution of shadow patterns visible in the MW camera image (Figure 6b) agree with the digital camera image (Figure 6a).
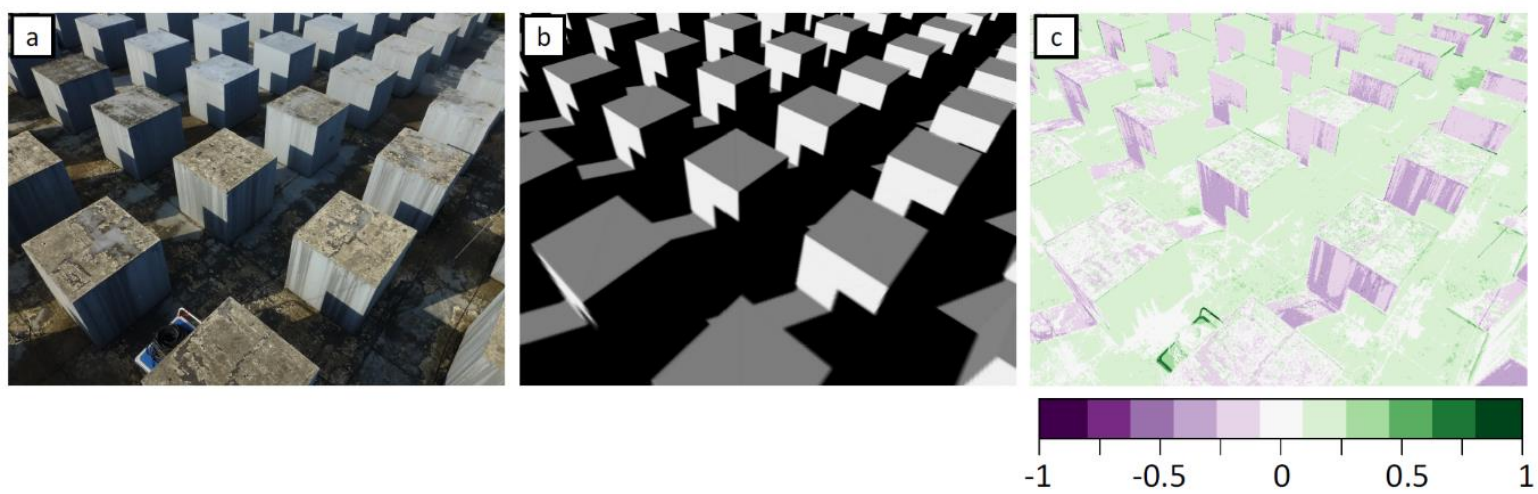

Figure 6. Images of COSMO test site taken at 15:25 local standard time on $26^{\text {th }}$ September 2014 with approximate $C^{\text {north }}$ perspective (Figure 2) from (a) a 'real world' $(R W)$ digital camera and (b) simulated by a 'model world' $(M W)$ camera in the shortwave using the Discrete Anisotropic Radiative Transfer (DART) model to compare the performance of DART when 1) simulating RW camera perspectives 2) resolving shadow patterns at high spatial resolution $(0.04 \mathrm{~m})$ across a canopy surface. Greyscale intensity (b) is used for qualitative indication of shaded (black) and sunlit (grey $\rightarrow$ white) surfaces. Comparison (c) shown as $R W-M W$ greyscale intensity difference.

\section{Longwave radiation flux extrapolated to 3D distribution}

Data from classified images (Section 2.4.3) are used with the classified MW surface (Section 2.3.2) to produce $M_{L W}^{3 D}$ at high spatial resolution. Pixels classified as class $i(x, y)$ within each MW camera image are associated with observations from the RW LWIR camera to obtain classified RW $M_{L W}^{c a m}(x, y)$ in the form $M_{L W, i}^{c a m}(x, y)$. The mixed and masked pixels (Figure $5 \mathrm{~b}, \mathrm{f}$ ) are not considered. All pixels for a given class from all cameras are aggregated to a mean value $\widetilde{M}_{L W, i}^{c a m}$. Voxels of class $i$ are assigned $M_{L W, i}^{c a m}$ to resolve per-voxel $M_{L W}$, shown in Figure 7 as brightness temperatures. This product constitutes $M_{L W}^{3 D}$ for a given time step. A voxel may be intersected by two or more triangles with faces of different class, which can occur at the corner of a building (Figure 7). In this case, the mean of $\widetilde{M}_{L W, i}^{c a m}$ for all classes involved is calculated for these voxels. This causes the unique brightness temperature values at intersecting facets with different orientation and temperature (Figure 7). 
The view angle configuration of the RW cameras (Figure 2) and the nature of allocatable surface classes means the classes assigned in $3 \mathrm{D}$ space $V x_{i}^{S}(\Delta X, \Delta Y, \Delta Z)$ are always observed by a camera for any given time step. The spatial form of $M_{L W}^{3 D}$ is inherently linked to the DSM (Figure 3 ) meaning the methodology is applicable to complex geometry and limited only by the voxel resolution and DSM level of detail.

Figure 7. Per-voxel brightness temperature $\left(V x_{T_{b}}^{S}\right)$ extrapolated from observations for one time-step $\left(2^{\text {nd }}\right.$ August 2014 10:00 local time, (same as Figure 5) for an arbitrary $8 \mathrm{~m} \times 8 \mathrm{~m}$ subset of the 'model world' $(M W)$ domain visualized as a three-dimensional point cloud, with each point at the centroid of a voxel. $V x_{T_{b}}^{S}(\Delta X, \Delta Y, \Delta Z)$ resolved at $\Delta X=\Delta Y=\Delta Z=0.04 \mathrm{~m}$ spatial resolution.

4. Results and discussion

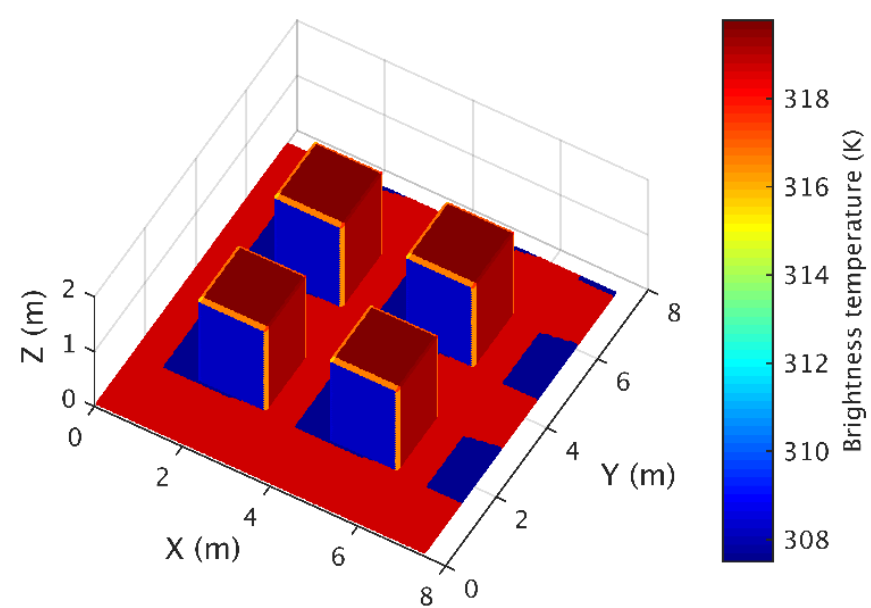

The methodology is applied using observations referenced at local time for $2^{\text {nd }}$ August 2014 (day of year 214). This is a mostly cloud-free day following an extended dry period of cloudy and part-cloudy days. The short time period is chosen to ensure the high temporal resolution of observations is fully applied and resolved.

\subsection{Image classification}

The classification methodology enables quantitative identification of the surface types seen by each camera on a per-pixel level. Figure 8 summarises the fraction of pixels assigned to each class within each camera image. Differences in the intercamera pixel fractions assigned to each class can be explained by the location and orientation of each camera. $\mathrm{C}^{\text {north }}$ views the higher fraction of ground and roof surfaces $(53.80 \%, 10327$ pixels) due to the lower camera zenith angle (Figure 2). Camera $\mathrm{C}^{\text {south }}$ views a higher fraction of vertical surfaces $\left(37.44 \%, 7190\right.$ pixels). Mixed pixels make up $29.12 \%$ (C ${ }^{\text {south }}$ ) and $20.11 \%\left(\mathrm{C}^{\text {north }}\right)$ of the images. Mixed pixels are identified during image classification of surface geometry (e.g. Figure $5 \mathrm{~b}$ ) and if any classified pixels are isolated (Section 2.4.3). $\mathrm{C}^{\text {south }}$ imagery contains $528(2.75 \%)$ masked radiometer boom pixels and more mixed pixels than $\mathrm{C}^{\text {north }}$. This is explained by its higher zenith angle and therefore longer average path length. Surfaces further away from the camera are more likely to be mixed within each pixel IFOV.

When a vertical surface first becomes insolated, the incident radiation is low as the angle of incidence is near parallel with the surface. In the MW, this is associated with a low density of DART illumination rays (Section 2.3.2) incident on these surfaces which introduces erroneous patterns in surface insolation status. Until the density of rays is sufficient across the MW surface, some pixels may be isolated from other pixels of the same insolation class. This explains the observed temporal variability in mixed pixels that coincides with walls coming into, and out of, shade. It could be corrected by increasing the surface density of illumination rays in the DART SW simulations (Section 2.3.2) at the expense of computation time. With the given MW resolution, the effect occurs twice for both cameras around 10:00 and 13:00. Each period has a $\sim 10$ min duration that increases mixed pixels across each image by up to $9 \%$ (Figure 8 ). Afternoon periods when all non-mixed pixels are intermittently classified as shaded are caused by short periods of overcast conditions based on direct incoming SW radiation (Figure 9a) measurements taken at the COSMO test site using a MS-56 Pyrheliometer (EKO Instruments). 


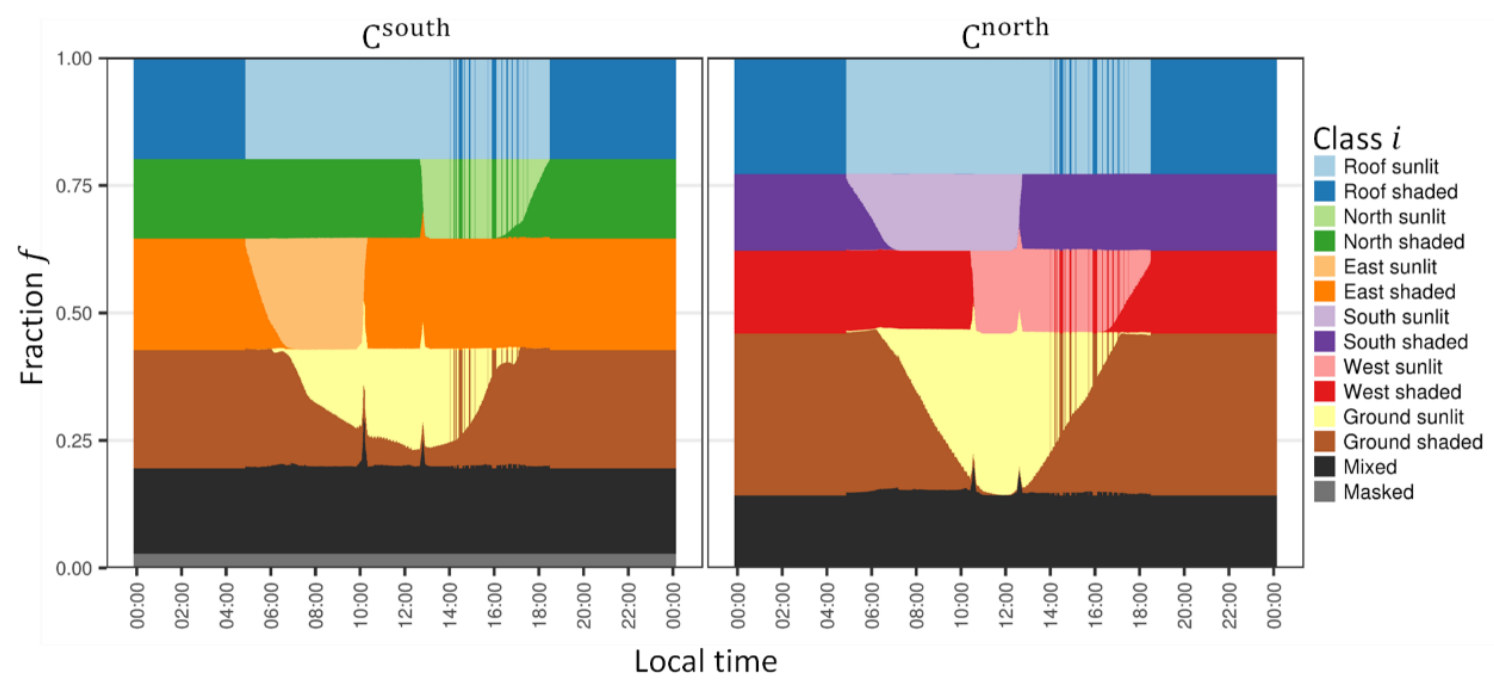

Figure 8. Fraction $f$ of all pixels in a camera image assigned to surface class $i$ for cameras $C^{\text {south }}$ and $C^{\text {north }}$ for day of year 214 at local standard time. Resolution of classified images is $1 \mathrm{~min}$. Short periods of daytime shadow due to overcast periods determined from $\left[E_{S W, \text { dir }}^{\downarrow}(\mathrm{COSMO})\right]$ observations (Figure 9). Mixed pixels contain more than one surface class or are isolated pixels (Section 2.4.3).

4.2. Inter-camera comparison

$M_{L W}^{c a m}$ agreement between instruments using manufacturer derived calibration coefficients (Section 2.2) is evaluated using measurements taken during the study day. Given the camera fields of view did not overlap, contact thermocouples were installed to give reference measurements enabling comparison of the camera calibrations. Unfortunately, instrumentation issues resulted in complete data loss from the thermocouples. Roof facets have a highly uniform radiative environment across the whole COSMO test site and offer the best available comparison to evaluate camera agreement (Figure 9). Camera heights and zenith angles are similar (Section 2.1 ) with $19.86 \%$ and $22.26 \%$ of image pixels classified as roofs for $\mathrm{C}^{\text {south }}$ and $\mathrm{C}^{\text {north }}$, respectively (Figure 8). Results show a systematic difference (slope 1.07, intercept $-22.01 \mathrm{~K}$ ) between observations of the roof pixels by the two cameras (Figure 9). The camera calibration sensitivity to camera body temperature in an outdoor setting is likely the primary contributor to the instrument uncertainty seen with the setup at COSMO $(2 \mathrm{~K})$. This translates into systematic differences in the observations and a hysteresis effect (Figure 9). Potentially rapid and uneven changes in temperature across each camera body cannot be fully accounted for by the camera calibration routine (Section 2.2). Other contributing factors, which cannot be easily quantified at this observational scale using the classification approach adopted, include differences in roof emissivity from surface weathering effects and anisotropy in surface emissivity (Nakayoshi et al., 2015). To reduce the impact of any systematic sensor disagreement on the extrapolated $M_{L W}^{3 D}$ product, $C^{\text {north }}$ is corrected to $C^{\text {south }}$ by linear regression using observations of the roof pixels from each camera (Figure 9). This approach is considered reasonable within the scope of the study as inter- and intra-facet variability is retained, and sensor specific biases are minimised.
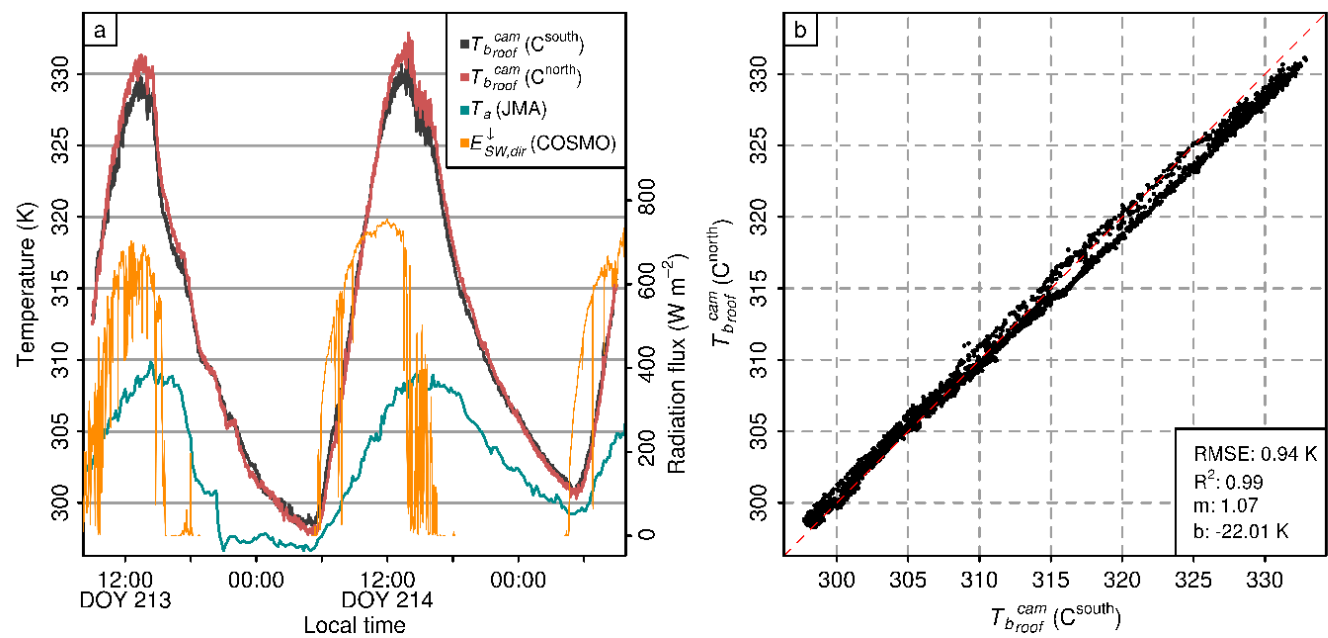

Figure 9. Inter-camera comparison of the mean broadband longwave radiation derived from roof pixels within the FOV of both cameras

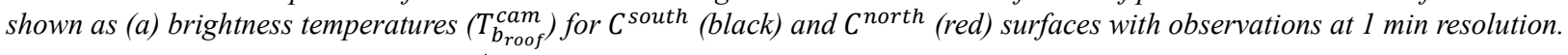
Direct incoming shortwave radiation $E_{S W}^{\downarrow}$,dir (COSMO)] measured on site using MS-56 Pyrheliometer (EKO Instruments) at 1 min resolution. Japan Meteorological Agency (JMA) air temperature measurements [ $T_{a}$ (JMA)] measured at Kumagaya AMeDAS (Automated Meteorological Data Acquisition System) station at 1 min resolution. (b) Relation between $T_{b_{\text {roof }}}^{\text {cam }}\left(C^{\text {south }}\right)$ and $T_{b_{\text {roof }}}^{\text {cam }}$ $\left(C^{\text {north }}\right)$ with linear regression slope $(m)$ and intercept (b) coefficients, used to correct the observations used throughout all results. In this figure observations are shown uncorrected. 


\subsection{Classified brightness temperature observations}

Variability of inter-class and intra-class observations is shown in Figure 10 on a per-pixel level for all cameras throughout the study date (mixed and masked pixels are excluded). As expected, the variability of the pre-classified pixels for all cameras (Figure 10a) is greatest during the daytime, with hourly differences between the $5^{\text {th }}$ and $95^{\text {th }}$ percentiles of pixel distributions reaching 18.4 K between 12:00 - 13:00. With increasing level of spatial detail in pixel classification accounting for insolation status (Figure 10b), orientation (Figure 10c) and both combined (Figure 10d), the inter-class ranges of $T_{b}^{c a m}$ typically decrease. This suggests the class related differences are helpful in explaining some of the $T_{b}^{c a m}$ variability. Brightness temperatures are most variable for the ground pixels (Figure 10c), with shaded or sunlit ground surface pixel distributions (Figure 10d) both being large throughout daytime. Hourly differences between $5^{\text {th }}$ and $95^{\text {th }}$ percentiles reach 15.0 K (12.2 K) for all shaded (sunlit) ground surface pixels between 12:00 - 13:00 (11:00 - 12:00). The shadowing history is associated with this variability, with a thermal hysteresis effect due to the thermal inertia of concrete. For example, between 14:00 - 15:00, the $25^{\text {th }}$ percentile of sunlit pixels have similar values to the $75^{\text {th }}$ percentile of shaded pixels. The greater sky view factor of roofs compared to all other facets influences inter-class variability, with median brightness temperature of roof pixels up to $2 \mathrm{~K}$ lower than all other classes between 00:00 - 05:00 (sunrise 04:55). Similarly, daytime roof brightness temperatures are highest (median $=330.8 \mathrm{~K}, 13: 00-14: 00$, Figure 10c) and for this time interval $15 \mathrm{~K}$ greater than north wall facet temperatures.

\subsection{Extrapolated longwave radiation flux}

The parameterisation of $M_{L W}^{3 D}$ is evaluated by modelling its upwelling LWIR radiation projected onto the IP of MW cameras. The per-pixel MW camera perspective is $M_{L W}^{c a m}(\mathrm{MW}, x, y)$ (i.e. Figure $\left.5 \mathrm{~d}, \mathrm{~h}\right) . M_{L W}^{c a m}(\mathrm{RW})$ is extrapolated to $M_{L W}^{3 D}$ (Section 3) with per-pixel differences calculated $\left[M_{L W}^{c a m}(\mathrm{RW}, x, y)-M_{L W}^{c a m}(\mathrm{MW}, x, y)\right]$ as an evaluation step. Figure 11 shows brightness temperature differences [i.e. $\left.T_{b}^{c a m}(\mathrm{RW}, x, y)-T_{b}^{c a m}(\mathrm{MW}, x, y)\right]$ at six times during the case study day. Nighttime period per-pixel RW - MW brightness temperature differences never exceed $\pm 1 \mathrm{~K}$ (not shown) due to the low intra-class variability (Figure 10d). Daytime per-pixel RW - MW differences are evident and indicate some RW processes remain unresolved by $M_{L W}^{3 D}$. Areas within the imagery where $T_{b}^{c a m}(\mathrm{MW})$ underestimates $T_{b}^{c a m}(\mathrm{RW})$ (red) or where $T_{b}^{c a m}(\mathrm{MW})$ overestimates $T_{b}^{\text {cam }}(\mathrm{RW})$ (blue) include edges of building blocks, edges of shadows and locations across all ground surfaces. $T_{b}^{c a m}(\mathrm{MW})$ typically underestimates $T_{b}^{c a m}(\mathrm{RW})$ for top-of-wall pixels. Absolute maximum differences between $T_{b}^{\text {cam }}(\mathrm{RW})$ and $T_{b}^{\text {cam }}(\mathrm{MW})$ can reach $15 \mathrm{~K}$ for individual pixels within both $\mathrm{C}^{\text {south }}$ and $\mathrm{C}^{\text {north }}$ imagery, with $1^{\text {st }}$ and $99^{\text {th }}$ percentiles $-4.34 \mathrm{~K}$ and $4.97 \mathrm{~K}$, respectively. There are artefacts in MW camera imagery around all roof edges that face away from the cameras (e.g. Figure $5 \mathrm{~d}, \mathrm{~h}$ ). These are caused by the resolution of $M_{L W}^{3 D}$ and DART discretisation of LWIR surface exitance and explain some of the large absolute differences around roof edge pixels. The camera point spread function may impact how well the intersection between facets of contrasting temperatures are resolved but was not available from the manufacturer for further investigation. This effect coupled with any slight misalignment between RW and MW cameras (Section 2.4.2) may compound to explain high $T_{b}^{c a m}(\mathrm{RW})-T_{b}^{c a m}(\mathrm{MW})$ differences near facet edges. 


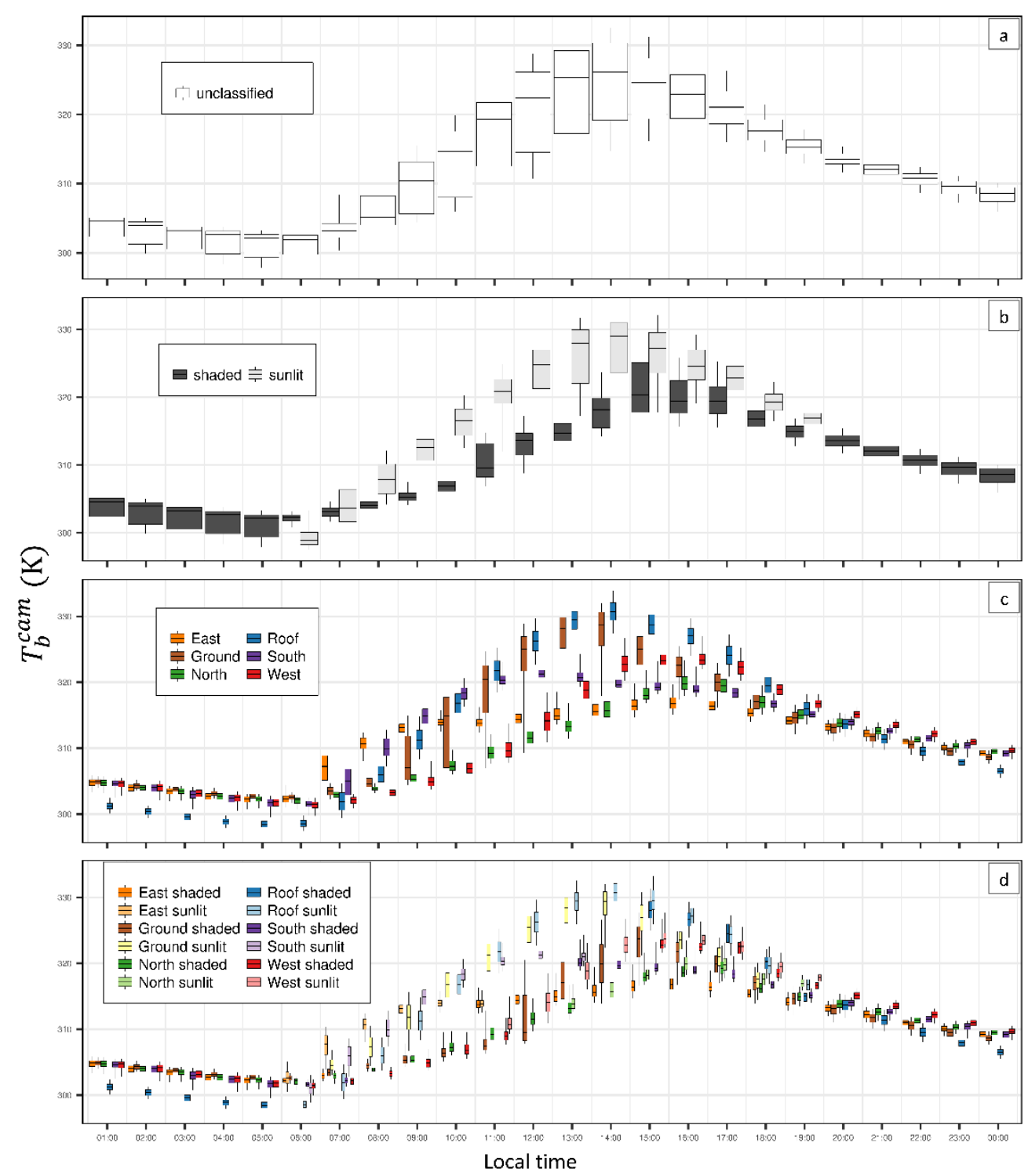

Figure 10. Intra-class variability of camera brightness temperatures $\left(T_{b}^{\text {cam }}\right)$ on $2^{\text {nd }}$ August 2014. Each boxplot is all pixels assigned to a class (colour) from both cameras (images 1 min samples) during $1 \mathrm{~h}$, with 5 and 95 percentiles (whiskers), interquartile range (box) and median (horizontal line) for pixels classified by: (a) all, (b) surface insolation status, (c) surface orientation, and (d) orientation and insolation status.

Surface energy exchange processes may further contribute to $T_{b}^{c a m}(\mathrm{RW})-T_{b}^{c a m}(\mathrm{MW})$ differences near facet edges. The building blocks are hollow causing different thermal admittance at their edges. Further, the edges of buildings may be exposed to higher wind speeds which modify heat transfer and therefore surface temperature. In addition, roof edges on the sunlit side of buildings have distinctly high observed brightness temperatures (e.g. Figure 5a, e) associated with the different sky view factors. Maximum brightness temperatures for mixed pixels at these building edges are up to $7.4 \mathrm{~K}$ (13.3 K) higher than the median of intersecting roof (wall) facets at 13:35. This may be explained by high solar irradiance (high sky view factor) and absorption of these areas throughout the day.

Closer to the ground, wall view factors are larger and ground surface reflection captured by $T_{b}^{c a m}(\mathrm{RW})$ is more important. Surfaces in these regions receive radiation from regions with cooler surface temperatures that have been in shade for prolonged periods (e.g. Figure 11e), and from regions with warmer surface temperatures that have been sunlit for prolonged periods (e.g. Figure 11i). The large distribution of brightness temperatures for observed ground pixels (Figure $10 \mathrm{c}, \mathrm{d}$ ) is not represented in $M_{L W}^{3 D}$ which only contains information on mean values of $M_{L W, i}^{c a m}$ (Section 3). The errors associated with this assumption are seen in Figure 11 mainly for shaded ground pixels and ground pixels at the edges of buildings. A shadow hysteresis is evident at multiple time steps (e.g. Figure $11 \mathrm{~d}$ center block, Figure $11 \mathrm{i}$ front center block) as brightness temperatures of surfaces coming out of (into) shade are overestimated (underestimated) by $M_{L W}^{3 D}$.

The extent to which these unresolved sub-facet processes influence the directional brightness temperature aggregated across each IP of $T_{b}^{c a m}(M W)$ is subsequently investigated. The aggregated at-sensor brightness temperature $\left(\widetilde{T}_{b}^{\text {cam }}\right)$ for $T_{b}^{c a m}(R W)$ and $T_{b}^{c a m}(M W)$ view is determined at 15 min resolution for the whole day ( 
Morrison W, S Kotthaus, CSB Grimmond, A Inagaki, T Yin, J-P Gastellu-Etchegorry, M Kanda, CJ Merchant 2018: A novel method to obtain three-dimensional urban surface temperature from ground-based thermography Remote Sensing of the Environment https://doi.org/10.1016/j.rse.2018.05.004 
Morrison W, S Kotthaus, CSB Grimmond, A Inagaki, T Yin, J-P Gastellu-Etchegorry, M Kanda, CJ Merchant 2018: A novel method to obtain three-dimensional urban surface temperature from ground-based thermography Remote Sensing of the Environment https://doi.org/10.1016/j.rse.2018.05.004

Figure 12). $\widetilde{T}_{b}^{c a m}$ is the mean of all non-masked pixels in a camera IP. This analysis is similar to results demonstrated for existing sensor view modelling approaches (e.g. Soux et al., 2004). In this paper, $\tilde{T}_{b}^{\text {cam }}(R W)-\tilde{T}_{b}^{c a m}(M W)$ differences show a diurnal pattern ( 
Morrison W, S Kotthaus, CSB Grimmond, A Inagaki, T Yin, J-P Gastellu-Etchegorry, M Kanda, CJ Merchant 2018: A novel method to obtain three-dimensional urban surface temperature from ground-based thermography Remote Sensing of the Environment https://doi.org/10.1016/j.rse.2018.05.004

Figure 12). When $M_{L W}^{3 D}$ is prescribed using insolation and orientation (solid lines, 
Morrison W, S Kotthaus, CSB Grimmond, A Inagaki, T Yin, J-P Gastellu-Etchegorry, M Kanda, CJ Merchant 2018: A novel method to obtain three-dimensional urban surface temperature from ground-based thermography Remote Sensing of the Environment https://doi.org/10.1016/j.rse.2018.05.004

Figure 12), RW - MW differences reach $\pm 0.65 \mathrm{~K}$ during daytime at $12: 45$ for $C^{\text {north }}$, and are within $0.40 \mathrm{~K}$ during nighttime. Prescribing $M_{L W}^{3 D}$ based only on orientation (dashed lines, 
Morrison W, S Kotthaus, CSB Grimmond, A Inagaki, T Yin, J-P Gastellu-Etchegorry, M Kanda, CJ Merchant 2018: A novel method to obtain three-dimensional urban surface temperature from ground-based thermography Remote Sensing of the Environment https://doi.org/10.1016/j.rse.2018.05.004

Figure 12; shown as inter-class distributions at pixel level in Figure 10c) leads to good agreement at night when inter-class variability is small and shadows do not occur. During daytime, however, not accounting for shadow patterns means $\tilde{T}_{b}^{\text {cam }}(M W)$ is up to $0.90 \mathrm{~K}$ warmer (cooler) than $\tilde{T}_{b}^{\text {cam }}(R W)$ for $C^{\text {south }}\left(C^{\text {north }}\right)$ at 12:45.
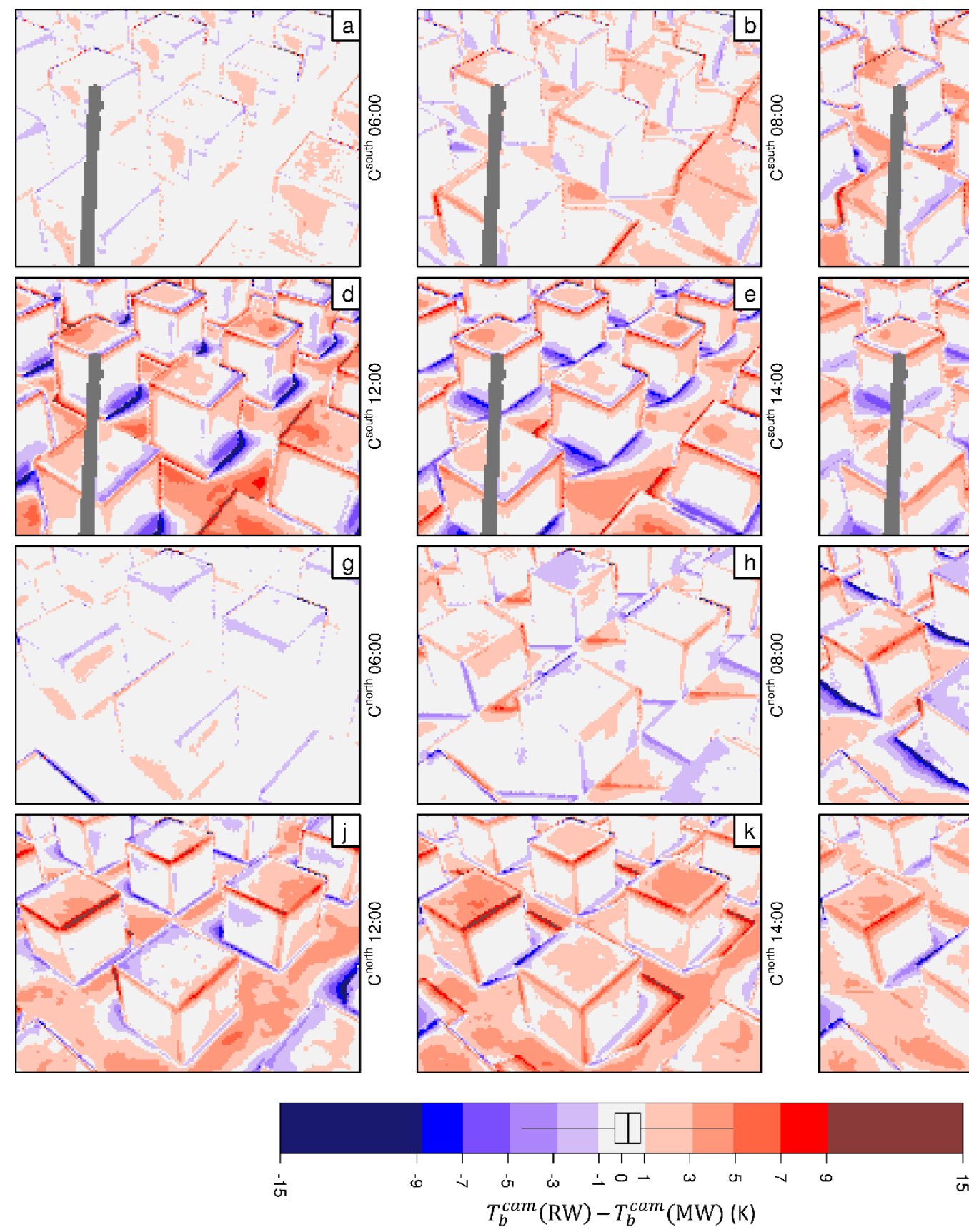

Figure 11. Brightness temperature $\left(T_{b}\right)$ differences between LWIR camera observations from 'real world' $(R W)\left[T_{b}^{c a m}(R W)\right]$ and 'model world' (MW) for six times (see labels on sub-plots) on $2^{\text {nd }}$ August 2014, for camera $(a-f) C^{\text {south }}$ and $(g-l) C^{\text {north }} . T_{b}^{\text {cam }}(M W)$ is simulated by DART camera view modelling using predetermined $3 D$ distribution of longwave flux $\left(M_{L W}^{3 D}\right)$. ( $\left.a-f\right)$ Radiometer boom masked (grey) from results. Boxplot (inside legend) for all non-masked pixels within all time steps throughout the day (15 min resolution) with $1^{\text {st }}$ and $99^{\text {th }}$ percentiles (whiskers), interquartile range (box) and median (horizontal line). 
Figure 12. Comparison of 'real world' $(R W)$ and 'model world' $(M W)$ aggregated at-sensor broadband longwave radiation observations (as brightness temperatures) for cameras $C^{\text {south }}$ (black) and $C^{\text {north }}$ (red) $2^{\text {nd }}$ August 2014 local standard time. MW observations simulated by DART camera view modelling using predetermined $3 D$ distribution of longwave flux $\left(M_{L W}^{3 D}\right)$ at the COSMO test site. $M_{L W}^{3 D}$ prescribed using all surface classes (solid lines) and orientation classes only (dashed lines).

\subsection{Normalised effective anisotropy}

Apparent brightness temperatures viewed from the COSMO surface over multiple discrete directions (375 directions over the hemisphere) are simulated using DART and $M_{L W}^{3 D}$. The apparent brightness temperature for a direction is the parallel projection of $M_{L W}^{3 D}$ onto a plane that is perpendicular to the view direction, aggregated to a single directional brightness temperature value. Here a $29.5 \mathrm{~m}$ x $29.5 \mathrm{~m}$ sample of $M_{L W}^{3 D}$ is analysed. The normalized effective anisotropy (Figure 13) is defined as the apparent brightness temperature from a nadir view minus the apparent brightness temperature at a given direction. Modelled values of normalised effective anisotropy range from $-6.10 \mathrm{~K}(12: 30)$ to $3.41 \mathrm{~K}(08: 00)$ on the case study day, with a maximum difference between any direction of $6.18 \mathrm{~K}$ (13:00). Lowest directional brightness temperatures occur at high zenith angles and at azimuth angles near the sun position. This is in agreement with prior results (Voogt, 2008). Around midday and early afternoon (e.g. 12:00, 14:00), view angles with high zenith angles $(\phi>$ $40^{\circ}$ ) near each cardinal azimuth angle underestimate nadir view brightness temperature by up to $6 \mathrm{~K}$. This is likely caused by the cooler walls occluding the warm ground surfaces at these view angles. A "hot spot" around the sun angle is prominent during morning periods $(08: 00,10: 00)$ where brightness temperature differences between currently insolated facets and shaded facets is greatest. Inter-facet temperature differences are lower during afternoon, reducing the magnitude of any hot spot $(14: 00,16: 00)$. These examples highlight a critical application of the modelling approach presented to any thermal RS study in a real urban setting.
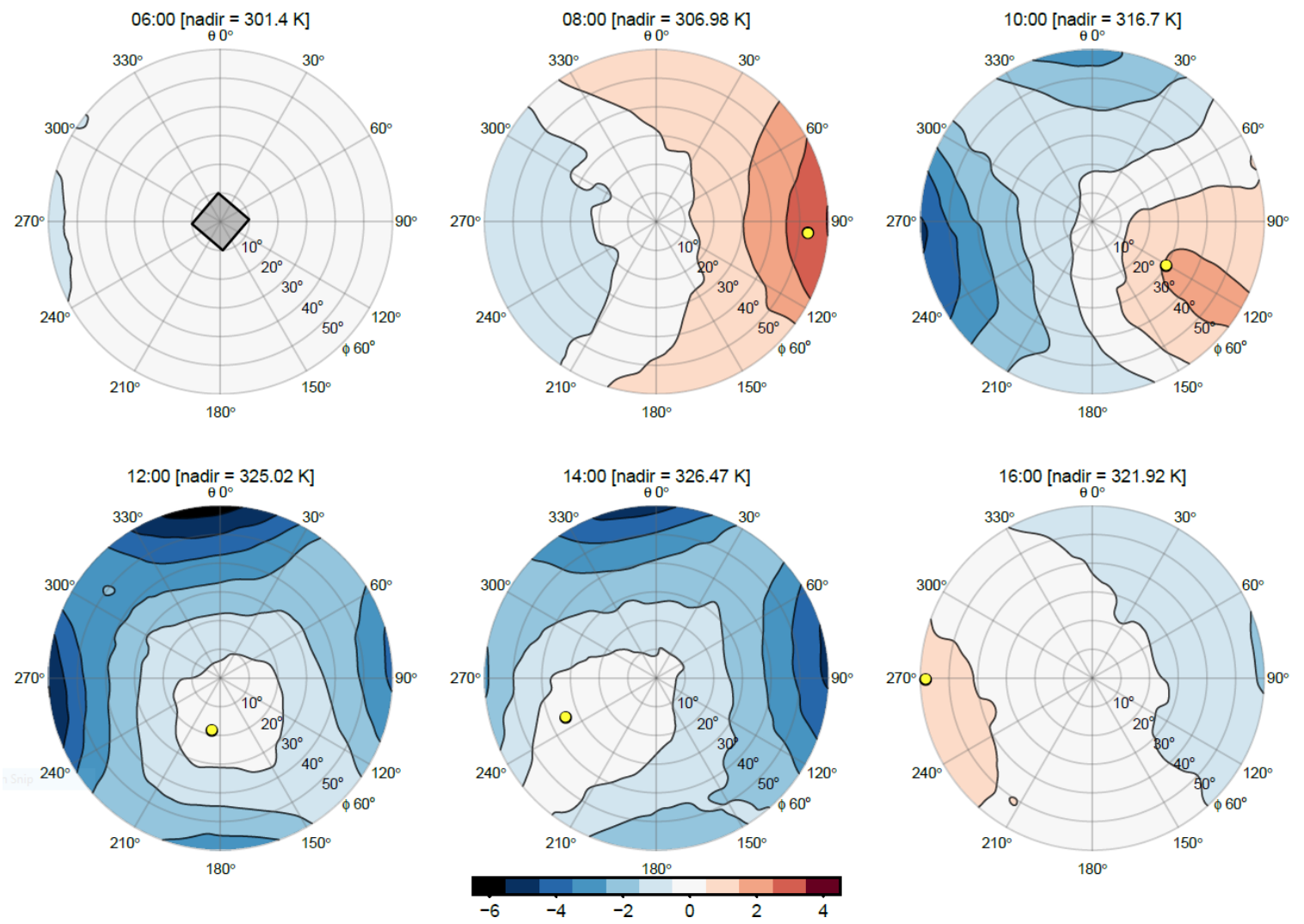

Figure 13. Simulated brightness temperatures for 375 discrete directions using predetermined 3D distribution of longwave flux $\left(M_{L W}^{3 D}\right)$ for the COSMO test site for 6 times on $2^{\text {nd }}$ August 2014. Results normalized by the simulated brightness 
Morrison W, S Kotthaus, CSB Grimmond, A Inagaki, T Yin, J-P Gastellu-Etchegorry, M Kanda, CJ Merchant 2018: A novel method to obtain three-dimensional urban surface temperature from ground-based thermography Remote Sensing of the Environment https://doi.org/10.1016/j.rse.2018.05.004

temperature at nadir $\left(\phi=0^{\circ}\right.$, given in sub-headings) and shown with $1 \mathrm{~K}$ contours. Data and sun angle (yellow dot) are oriented with true north $\left(\theta=0^{\circ}\right)$, with COSMO building orientation displayed (center grey square, 06:00).

\section{Conclusions}

The exitant longwave radiation from a simplified urban surface (COSMO outdoor scale model) is studied based on ground-based LWIR camera observations with detailed radiative transfer and camera view modelling. Ground based LWIR camera $\left(M_{L W}^{c a m}\right)$ observations from the COSMO test site over $24 \mathrm{~h}$ illustrate spatial and temporal patterns in upwelling LWIR radiation.

Hourly per-pixel camera brightness temperatures are low at night, but vary more widely during the day $\left(5^{\text {th }}-95^{\text {th }}\right.$ percentile differences reach 313.05 - $331.45 \mathrm{~K}$ between 12:00 - 13:00). As shadows cast by buildings and facet orientations likely explain most of this observed variability, these are often used to parameterise kinematic and radiometric temperature variability across the complete urban surface. Here, radiative transfer and camera view modelling is undertaken to identify the sunlit/shaded dynamics of camera pixels. The nature of inter-class and intra-class $M_{L W}^{c a m}$ distributions derived from the modelling results suggest that manual digitization or frequency distribution analysis may be problematic, particularly during daytime when inter-class distributions frequently overlap. This effect is most prominent for distributions of sunlit and shaded ground pixels.

Despite the simple surface geometry of the study site, image classification by camera view modelling demands small margins of error for camera parameters such as image distortion (Section 2.4.1) and the physical positioning of cameras (Section 2.4.2) for the perspective of $M_{L W}^{c a m}$ to be modelled accurately. This finding can be attributed to the short surfacesensor path lengths of the observational setup. Furthermore, sun-surface geometry and the timekeeping of observations must be known to a high degree of accuracy. Previous studies have not demonstrated direct comparison of observed and simulated results in such detail.

Methods in this paper overcome sensor view modelling challenges by using the DART and Blender camera view models. A comparison between a captured and modelled high resolution digital camera image (Section 2.4.3) demonstrates the potential accuracy and resolution of the methods. Evaluating the ability of classified $M_{L W}^{c a m}$ observations to explain the variability of broadband longwave radiation exitant across the COSMO canopy surface uses the 3D distribution of exitant LWIR radiation $\left(M_{L W}^{3 D}\right)$. It is concluded that $M_{L W}^{3 D}$ accurately resolves the surface geometry of the test site when the parameterisation is at high temporal $(15 \mathrm{~min})$ and spatial $(0.04 \mathrm{~m})$ resolution. $M_{L W}^{3 D}$ is evaluated for a given time step by projecting prescribed values of exitant broadband longwave radiation onto simulated 'model world' (MW) cameras with perspectives matching that of the 'real world' (RW) $M_{L W}^{c a m}$. Pixel level comparison between RW and MW camera imagery identifies areas where the prescribed $M_{L W}^{3 D}$ does not resolve $M_{L W}^{c a m}$ variability; viz, building edges, sky view factor variability of vertical surfaces, and ground areas with a distinct shadow hysteresis. By aggregating all pixels in each image to a single brightness temperature for each MW and RW camera, these features average out to RW - MW differences within $0.65 \mathrm{~K}$ throughout a $24 \mathrm{~h}$ period. Understanding unresolved sub-facet processes may be required for parameterisation of $M_{L W}^{c a m}$ in more complex urban environments. Further classes of absolute irradiance values and sky view factor have potential to be applied using DART.

$M_{L W}^{3 D}$ coupled with DART is shown to be a useful for assessment of urban thermal anisotropy (Section 4.5). Modelled anisotropy results for the study day show large differences between nadir and off-nadir apparent brightness temperatures which in general is in good agreement with prior studies. Modelled anisotropy is simplified in that it does not consider irregular building geometry and materials found in real cities. The regularity of the COSMO geometry may introduce an overestimation of modelled anisotropy compared to real world cities (Krayenhoff and Voogt, 2016).

It is concluded that shadow histories classes and further quantification of surface irradiance fluxes using DART radiative transfer functionality would be useful to address in future studies. Depending on the availability of spatial databases, building geometry and material properties can also be resolved at levels of detail representative of complex urban environments. Material properties may be challenging to obtain due to a general lack of urban materials spatial databases. If the surface form is a predominant factor in effective thermal anisotropy, simplified assumptions of surface material may be sufficient when classifying $M_{L W}^{c a m}$ and parameterising $M_{L W}^{3 D}$ for complex urban environments.

Applying the methods presented in this paper to real cities may require a different approach to sensor siting. The current study uses a mast as a sensor platform that is $\sim 4$ times the mean building height (Figure 2 ). This enables an observational source area that is representative of the domain and that resolves sub-facet processes. A similar source area could be achieved in urban areas with compact low-rise and open high-rise morphology, with cameras installed on top of the taller high-rise buildings.

Overall, this work provides a significant improvement to interpreting ground-based RS observations. Applied to real city settings, this has the potential to provide essential improvements to evaluating errors associated with operationally retrieved urban surface temperatures from satellite RS platforms and the parameterisation of longwave radiation exchanges in urban surface schemes. 
Morrison W, S Kotthaus, CSB Grimmond, A Inagaki, T Yin, J-P Gastellu-Etchegorry, M Kanda, CJ Merchant 2018: A novel method to obtain three-dimensional urban surface temperature from ground-based thermography Remote Sensing of the Environment

\section{Acknowledgements}

This work is funded by Newton Fund/Met Office Climate Science for Service Partnership China (CSSP China), EU H2020 UrbanFluxes, Japan Society for the Promotion of Science (JSPS), NERC ClearfLo, NERC/Belmont TRUC, EUf7 emBRACE and the National Research Foundation (NRF) Singapore through the Singapore-MIT Alliance for Research and Technology's Centre for Environmental Sensing and Modeling (SMART-CENSAM) interdisciplinary research program. We thank Nicholas Lauret and Lucas Landier of the DART team for ongoing DART model development and valuable support. We thank all students at Kanda lab who contributed to conducting the measurements.

\section{References}

Adderley, C., Christen, A., Voogt, J.A., 2015. The effect of radiometer placement and view on inferred directional and hemispheric radiometric temperatures of a urban canopy. Atmos. Meas. Tech. Discuss. 8, 1891-1933. https://doi.org/10.5194/amtd-8-1891-2015

Bouguet, J.-Y., 2015. Camera calibration toolbox for Matlab [WWW Document]. http://www.vision.caltech.edu/bouguetj/calib_doc/

Budzier, H., Gerlach, G., 2015. Calibration of uncooled thermal infrared cameras. J. Sensors Sens. Syst. 4, $187-197$. https://doi.org/10.5194/jsss-4-187-2015

Christen, A., Meier, F., Scherer, D., 2012. High-frequency fluctuations of surface temperatures in an urban environment. Theor. Appl. Climatol. 108, 301-324. https://doi.org/10.1007/s00704-011-0521-x

Clarke, T.A., Wang, X., Fryer, J.G., 1998. The Principal Point and CCD Cameras. Photogramm. Rec. 16, $293-312$. https://doi.org/10.1111/0031-868X.00127

Gastellu-Etchegorry, J.-P., Grau, E., Lauret, N., 2012. DART: A 3D model for remote sensing images and radiative budget of earth surfaces.

Gastellu-Etchegorry, J.P., 2008. 3D modeling of satellite spectral images, radiation budget and energy budget of urban landscapes. Meteorol. Atmos. Phys. 102, 187-207. https://doi.org/10.1007/s00703-008-0344-1

Gastellu-Etchegorry, J.P., Yin, T., Lauret, N., Cajgfinger, T., Gregoire, T., Grau, E., Feret, J.B., Lopes, M., Guilleux, J., Dedieu, G., Malenovskỳ, Z., Cook, B.D., Morton, D., Rubio, J., Durrieu, S., Cazanave, G., Martin, E., Ristorcelli, T., 2015. Discrete anisotropic radiative transfer (DART 5) for modeling airborne and satellite spectroradiometer and LIDAR acquisitions of natural and urban landscapes. Remote Sens. 7, 1667-1701. https://doi.org/10.3390/rs70201667

Hartley, R.I., Zisserman, A., 2000. Multiple View Geometry in Computer Vision. Cambridge University Press, ISBN: 0521623049. https://doi.org/10.1017/S0263574705211621

Heikkila, J., Silven, O., 1993. A four-step camera calibration procedure with implicit image correction, in: Proceedings of IEEE Computer Society Conference on Computer Vision and Pattern Recognition. IEEE Comput. Soc, pp. 1106-1112. https://doi.org/10.1109/CVPR.1997.609468

Huang, F., Zhan, W., Voogt, J., Hu, L., Wang, Z., Quan, J., 2016. Remote Sensing of Environment Temporal upscaling of surface urban heat island by incorporating an annual temperature cycle model : A tale of two cities. Remote Sens. Environ. 186, 1-12. https://doi.org/10.1016/j.rse.2016.08.009

Johnson, G.T., Watson, I.D., 1984. The Determination of View-Factors in Urban Canyons. J. Clim. Appl. Meteorol. 23, $329-335$. https://doi.org/10.1175/1520-0450(1984)023<0329:TDOVFI $>2.0 . C O ; 2$

Kanda, M., Kanega, M., Kawai, T., Moriwaki, R., Sugawara, H., 2007. Roughness lengths for momentum and heat derived from outdoor urban scale models. J. Appl. Meteorol. Climatol. 46, 1067-1079. https://doi.org/10.1175/JAM2500.1

Kato, S., Yamaguchi, Y., 2005. Analysis of urban heat-island effect using ASTER and ETM+ Data: Separation of anthropogenic heat discharge and natural heat radiation from sensible heat flux. Remote Sens. Environ. 99, 44-54. https://doi.org/10.1016/j.rse.2005.04.026

Krayenhoff, E.S., Voogt, J.A., 2007. A microscale three-dimensional urban energy balance model for studying surface temperatures. Boundary-Layer Meteorol. 123, 433-461. https://doi.org/10.1007/s10546-006-9153-6

Krayenhoff, S., Voogt, J., 2016. Daytime thermal anisotropy of urban neighbourhoods: Morphological causation. Remote Sens. 8, 1-22. https://doi.org/10.3390/rs8020108

Kusaka, H., Kondo, H., Kikegawa, Y., Kimura, F., 2001. A Simple Single-Layer Urban Canopy Model For Atmospheric Models: Comparison With Multi-Layer And Slab Models. Boundary-Layer Meteorol. 101, 329-358. https://doi.org/10.1023/A:1019207923078

Lagouarde, J.P., Hénon, A., Kurz, B., Moreau, P., Irvine, M., Voogt, J., Mestayer, P., 2010. Modelling daytime thermal infrared directional anisotropy over Toulouse city centre. Remote Sens. Environ. 114, 87-105. https://doi.org/10.1016/j.rse.2009.08.012

Lagouarde, J.P., Moreau, P., Irvine, M., Bonnefond, J.M., Voogt, J.A., Solliec, F., 2004. Airborne experimental measurements of the angular variations in surface temperature over urban areas: Case study of Marseille (France). Remote Sens. Environ. 93, 443-462. https://doi.org/10.1016/j.rse.2003.12.011

Meier, F., Scherer, D., Richters, J., Christen, A., 2011. Atmospheric correction of thermal-infrared imagery of the 3-D urban environment acquired in oblique viewing geometry. Atmos. Meas. Tech. 4, 909-922. https://doi.org/10.5194/amt-4-909-2011

Nakayoshi, M., Kanda, M., de Dear, R., 2015. Globe Anemo-radiometer. Boundary-Layer Meteorol. 155, $209-227$. https://doi.org/10.1007/s10546-014-0003-7

Roth, M., Oke, T.R., Emery, W.J., 1989. Satellite-derived urban heat islands from three coastal cities and the utilization of such data in urban climatology. Int. J. Remote Sens. 10, 1699-1720. https://doi.org/10.1080/01431168908904002

Schaepman-Strub, G., Schaepman, M.E., Painter, T.H., Dangel, S., Martonchik, J. V., 2006. Reflectance quantities in optical remote sensing-definitions and case studies. Remote Sens. Environ. 103, 27-42. https://doi.org/10.1016/j.rse.2006.03.002

Soux, A., Voogt, J.A., Oke, T.R., 2004. A model to calculate what a remote sensor "sees" of an urban surface. Boundary-Layer Meteorol. 111, 109-132. https://doi.org/10.1023/B:BOUN.0000010995.62115.46

Sugawara, H., Takamura, T., 2006. Longwave radiation flux from an urban canopy: Evaluation via measurements of directional radiometric temperature. Remote Sens. Environ. 104, 226-237. https://doi.org/10.1016/j.rse.2006.01.024

Thorsson, S., Lindqvist, M., Lindqvist, S., 2004. Thermal bioclimatic conditions and patterns of behaviour in an urban park in Göteborg, Sweden. Int. J. Biometeorol. 48, 149-156. https://doi.org/10.1007/s00484-003-0189-8

Vollmer, M., Möllmann, K.-P., 2010. Infrared Thermal Imaging. Wiley-VCH Verlag GmbH \& Co. KGaA, Weinheim, Germany. https://doi.org/10.1002/9783527630868 
Morrison W, S Kotthaus, CSB Grimmond, A Inagaki, T Yin, J-P Gastellu-Etchegorry, M Kanda, CJ Merchant 2018: A novel method to obtain three-dimensional urban surface temperature from ground-based thermography Remote Sensing of the Environment https://doi.org/10.1016/j.rse.2018.05.004

Voogt, J.A., 2008. Assessment of an Urban Sensor View Model for thermal anisotropy. Remote Sens. Environ. 112, $482-495$. https://doi.org/10.1016/j.rse.2007.05.013

Voogt, J.A., Grimmond, C.S.B., 2000. Modeling Surface Sensible Heat Flux Using Surface Radiative Temperatures in a Simple Urban Area. J. Appl. Meteorol. 39, 1679-1699. https://doi.org/10.1175/1520-0450-39.10.1679

Voogt, J.A., Oke, T.R., 2003. Thermal remote sensing of urban climates 86, 370-384. https://doi.org/10.1016/S0034-4257(03)00079-8

Voogt, J.A., Oke, T.R., 1998. Effects of urban surface geometry on remotely-sensed surface temperature. Int. J. Remote Sens. 19, 895920. https://doi.org/10.1080/014311698215784

Voogt, J. a., Oke, T.R., 1997. Complete Urban Surface Temperatures. J. Appl. Meteorol. 36, 1117-1132. https://doi.org/10.1175/15200450(1997)036<1117:CUST >2.0.CO;2

Xu, W., Wooster, M.J., Grimmond, C.S.B., 2008. Modelling of urban sensible heat flux at multiple spatial scales: A demonstration using airborne hyperspectral imagery of Shanghai and a temperature-emissivity separation approach. Remote Sens. Environ. 112, 34933510. https://doi.org/10.1016/j.rse.2008.04.009

Yin, T., Gastellu-Etchegorry, J.P., Lauret, N., Grau, E., Rubio, J., 2013. A new approach of direction discretization and oversampling for 3D anisotropic radiative transfer modeling. Remote Sens. Environ. 135, 213-223. https://doi.org/10.1016/j.rse.2013.03.030

Yin, T., Lauret, N., Gastellu-Etchegorry, J.-P., 2015. Simulating images of passive sensors with finite field of view by coupling 3-D radiative transfer model and sensor perspective projection. Remote Sens. Environ. 162, 169-185.

https://doi.org/10.1016/j.rse.2015.02.020 